\title{
4. Völkisches Denken in Publikationen ideologisch wahlverwandter Professoren. Drei Fallbeispiele
}

Ehe dem komplexen und facettenreichen Verhältnis Grimms, Kolbenheyers und Stapels zum Nationalsozialismus seit Beginn der 1920er Jahre bis zum Ende des Zweiten Weltkriegs nachgegangen wird, soll im Folgenden zunächst an drei Fallbeispielen gezeigt werden, inwiefern die zahlreichen, mit Grimm, Kolbenheyer und Stapel in unterschiedlicher Weise in Beziehung stehenden Hochschullehrer in ihren eigenen Publikationen und Vorträgen auf völkisches Gedankengut rekurrierten und somit zu dessen Verbreitung in der Gesellschaft der Weimarer Republik beitrugen. Ausgewählt wurden hier der Philosoph und Direktor des Instituts für experimentelle Psychologie an der Universität Leipzig Felix Krueger, der an der Universität Münster tätige Strafrechtslehrer Andreas Thomsen sowie der Tübinger Professor für Neuere Geschichte Adalbert Wahl.

Diese Auswahl illustriert nicht nur beispielhaft die Mannigfaltigkeit der wissenschaftlichen Disziplinen der weltanschaulich wahlverwandten Professoren, sondern zugleich die große Verschiedenartigkeit ihrer jeweiligen Beziehungen zu den Hauptprotagonisten der vorliegenden Untersuchung: Während Felix Krueger als Familienfreund Wilhelm Stapels beispielhaft für den Typus einer engen und vertrauensvollen Beziehung steht, repräsentiert der in Erwin Guido Kolbenheyers Wahlheimat lehrende Adalbert Wahl eine auf geografischer Nähe und regelmäßigem Umgang basierende Verbindung. Andreas Thomsen steht hingegen für den Beziehungstyp einer nur kurze Zeit dauernden, flüchtigen Bekanntschaft, namentlich mit Hans Grimm.

\subsection{Felix Krueger und die "Selbstbesinnung in deutscher Not"}

Inzwischen hat uns Deutsche die harte Schule unserer Geschichte auf das deutlichste erkennen gemacht; jenes hohe, uns gemäße Ziel können wir nur erreichen, wenn wir uns festigen und vertiefen in dem Glauben an den unersetzlichen Eigenwert unseres Volkstums. Dazu gehört, daß jeder zunächst einmal seine angestammte Eigenart treu bewahre, daß er in seinem Heimatboden starke Wurzeln habe und behalte. Nur aus diesen Kräften, naturgewachsener Gemeinschaft heraus, können wir hoffen, ganze Menschen zu werden. Dann aber wird uns das andere alles zufallen. ${ }^{1}$

Zur Biografie Kruegers - Felix Krueger, am 10.August 1874 als Sohn eines Fabrikanten in Posen geboren, studierte ab 1893 Philosophie, Psychologie, Ge-

1 Krueger, Verkehr, S. 25f. 
schichte, Wirtschaftswissenschaften und Naturwissenschaften mit dem Schwerpunkt Physik an den Universitäten Straßburg, München, Berlin und Leipzig. Nach seiner Promotion zum Thema Der Begriff des absolut Wertvollen als Grundbegriff der Moralphilosophie an der Universität München arbeitete er seit 1897 als Assistent des berühmten Psychologen Wilhelm Wundt² (1832-1920) am Leipziger Institut für experimentelle Psychologie. Seiner dortigen Habilitation im Jahr 1903 zum Thema Das Bewußtsein der Konsonanz. Eine psychologische Analyse folgten Ordinariate für angewandte Psychologie an der Universität Buenos Aires (19061908) sowie für Philosophie an der Universität Halle-Wittenberg seit 1910. ${ }^{3} \mathrm{Im}$ August 1914 meldete sich Krueger - ein ungewöhnlicher Schritt für einen annähernd vierzigjährigen Ordinarius - als Kriegsfreiwilliger. Krueger diente bis zum Juli 1917 und nahm unter anderem an der Schlacht von Verdun teil, ehe er im Oktober 1917 auf den Lehrstuhl für Philosophie an der Universität Leipzig sowie zum Nachfolger Wilhelm Wundts als Direktor des Instituts für experimentelle Psychologie berufen wurde. Zwischen 1927 und 1933 amtierte Krueger zudem als Vorsitzender der Deutschen Philosophischen Gesellschaft ${ }^{4}$, in den Jahren 1934 bis 1936 als Präsident der Deutschen Gesellschaft für Psychologie. Zeitgenössisch schlug sich das hohe wissenschaftliche Renommee Kruegers nicht zuletzt in Ehrendoktorwürden der New Yorker Columbia-Universität, des Wittenberg-College in Springfield/Ohio und der Technischen Hochschule Dresden nieder. ${ }^{5}$

Obgleich Krueger im Jahr 1933 das „Bekenntnis der Professoren an den deutschen Universitäten und Hochschulen zu Adolf Hitler" unterzeichnete, geriet er alsbald in Konflikt mit nationalsozialistisch orientierten Studenten seiner Universität. 1936 wurde Krueger, der im April 1935 das Rektorenamt der Universität Leipzig übernommen hatte, denunziert, nachdem er in einer seiner Vorlesung den Physiker Heinrich Hertz als „edlen Juden“ bezeichnet hatte. Empört über die Geistlosigkeit der Denunziation ergänzte Krueger daraufhin - nicht ohne Trotz in einer weiteren Vorlesung die Liste „edler Juden“ um den Komponisten Felix Mendelssohn Bartholdy, den Schriftsteller Paul Heyse und den niederländischen Philosophen Baruch de Spinoza. Vertreter des Nationalsozialistischer Deutscher Studentenbund (NSDStB) empfanden dies als unzumutbar und ungeachtet dessen, dass in Kruegers Distinktion zwischen vereinzelten „edlen“ und dem Gros „normaler" (ergo unedler) Juden bereits eine antisemitische Grundgesinnung zum Ausdruck $\mathrm{kam}^{6}$, führten die Äußerungen letztendlich zum Entzug seiner Lehrerlaubnis. Im März 1938 wurde er schließlich vorzeitig emeritiert. ${ }^{7}$ Krueger,

2 Vgl. Friedrich, Wundt.

3 Zu Kruegers Biografie und akademischen Werdegang bis 1914 vgl. Guski-Leinwand, Wissenschaftsforschung, S. 168-173.

4 Zur Geschichte der Deutschen Philosophischen Gesellschaft vgl. Tilitzki, Universitätsphilosophie, S. 473-544.

5 Vgl. UAL, Personalakte Felix Krueger (PA 664), Bl. 68.

6 Die seit 1933 geführte Debatte über vereinzelte verdienstvolle Juden hat Sebastian Haffner als Erfolg der Nationalsozialisten gewertet, die „Judenfrage“ im öffentlichen Diskurs zu etablieren. Vgl. Haffner, Geschichte, S. 138f.

7 Wolfradt, Ethnologie, S. 67. 
der unmittelbar vor Ende des Zweiten Weltkriegs in die Schweiz aussiedelte, starb am 25. Februar 1948 in Basel.

Die Bedeutung der psychologischen Ansätze Kruegers und seiner Schüler wird bis heute anerkannt. ${ }^{8}$ Die Forschung hat indes auch auf die prekäre ideologische Aufladung der maßgeblich von Krueger geprägten und vertretenen „Ganzheitspsychologie“ verwiesen: Der Begriff der „Ganzheit“ sei eine spezifische Reaktion auf „eine als Zerfall wahrgenommene politische und soziale Entwicklung “9 der deutschen Gesellschaft gewesen, an der Krueger den Verlust ihres einstmals angeblich „organischen“ Charakters diagnostizierte. Die maßgeblich von Krueger geformte und repräsentierte „Leipziger Schule“ der Ganzheitspsychologie grenzte sich scharf von der durch die Psychologen Max Wertheimer und Kurt Koffka vertretenen „Berliner Schule“ der „Gestalttheorie“ ab. Ihr wurde eine angeblich spezifisch jüdischen Denkweisen verpflichtete „Psychologie ohne Seele“ vorgeworfen - pauschal bemessen und umschrieben mit Schlagworten wie „materialistisch“, „rationalistisch“, „oberflächlich“ und „wurzellos“.10 Eine „Heilung der Zivilisationsschäden“ versprach sich Krueger in erster Linie durch die „Rück-Bindung des im Zivilisationsprozess atomisierten Individuums in transpersonale Ganzheiten“ und „ursprüngliche Gemeinschaften“, insbesondere in „Ehe, Familie, Sippe, Bünde, Volk“11. Die „Projektion der ,synthetisch-harmonisierenden Denkfigur“" auf das deutsche Volk als „handlungsfähig gedachte[s] Kollektiv“12, die Stefan Breuer treffend als ein zentrales Wesensmerkmal völkischen Denkens seit dem ausgehenden 19. Jahrhundert beschrieben hat ${ }^{13}$, ist hier mit Händen zu greifen. Sein gesellschaftliches Wunschmodell hielt Krueger indes unter dem Weimarer Parteiensystem für politisch nicht realisierbar.

Das Verhältnis Felix Kruegers zu Stapel und Kolbenheyer - Die Beziehung zwischen Krueger und Wilhelm Stapel war vielschichtig. Sie reichte von einem gemeinsamen Engagement in der Fichte-Gesellschaft seit den frühen 1920er Jahren $^{14}$ über die Mitarbeit Kruegers im Deutschen Volkstum ${ }^{15}$ und die Einladung Stapels als Redner auf einer Tagung der Deutschen Philosophische Gesellschaft ${ }^{16}$

${ }^{8}$ Unlängst wurden sie zu den „Meilensteinen“ in der Geschichte der Psychologie gerechnet. Vgl. Galliker/Klein/Rykart, Meilensteine, S. 304-317.

9 Tilitzki, Universitätsphilosophie, S. 528.

${ }^{10}$ Vgl. Harrington, Suche, S. $235 f$.

11 Ebd., S. $528 \mathrm{f}$.

12 Breuer, Völkischen, S. 17.

${ }^{13}$ Vgl. Kap. 1.2.

${ }^{14}$ Die im Juni 1919 von Felix Krueger in Leipzig gegründete Gesellschaft für deutsche Volksbildung wurde im Oktober 1920 zu einer Ortsgruppe der von Stapel maßgeblich beeinflussten Hamburger Fichte-Gesellschaft umgewandelt. Zur Gewährleistung einer engen Zusammenarbeit zwischen Leipzig und Hamburg wurde Krueger zum stellvertretenden Vorsitzenden der Fichte-Gesellschaft ernannt. Ein im Oktober 1920 gegründeter Schriftenausschuss der Fichte-Gesellschaft wurde von Krueger und Stapel gemeinsam mit Hans Gerber besetzt. Vgl. Mitteilungen der Fichte Gesellschaft von 1914, Blatt 4, o. O. 1921, S. 1.

${ }^{15}$ Vgl. Krueger, Okkultismus.

${ }^{16}$ Vgl. Stapel, Volk [1929]. 
bis hin zu einer Freundschaft auf familiärer Ebene. „Mit Krueger bin ich familiär befreundet“, schrieb Stapel im Oktober 1929 an Kolbenheyer. „Wir haben öfter bei Kruegers in Leipzig logiert, er und seine Frau bei uns"17. Informationen darüber, wann genau sich Stapel und Krueger erstmals kennenlernten, gehen jedoch weder aus dem Nachlass Stapels noch jenem Kruegers hervor.

Die erste Begegnung zwischen dem Leipziger Psychologen und Kolbenheyer lässt sich hingegen eindeutig datieren. Sie fand auf dem Geraer Dichtertreffen im Juni 1931 statt, von dem bereits die Rede gewesen ist. ${ }^{18}$ Kolbenheyer gewann auf der Tagung einen überaus positiven Eindruck von Krueger, wie er dem Psychologen im direkten Anschluss an die Tagung auch persönlich versicherte: „Wenn ich an die Geraer Tage zurückdenke, so fällt auf meine Begegnung und die Auszeichnung der Bekanntschaft mit Ihnen [...] eines der hellsten Strahlenbündel“19. Krueger selbst hatte sich bereits einige Jahre zuvor mit Kolbenheyers BauhüttenPhilosophie vertraut gemacht. Sein Fazit über das Werk bezeugt einerseits das grundsätzliche Interesse Kruegers an einer stärkeren Interdisziplinarität zwischen Biologie und Psychologie, andererseits jedoch auch seine Vorbehalte gegenüber Kolbenheyers augenfälliger Neigung zu monokausalem, biologistischem Denken. In diesem Vorbehalt kam Kruegers hohes Selbstbewusstsein als einer der damals führenden Vertreter seines mittlerweile traditionsreichen Fachgebiets zum Ausdruck. Eine Annäherung zwischen Psychologie und Biologie durfte laut Krueger nicht einseitig zu Ungunsten seiner Disziplin ausfallen, sondern musste wechselseitig erfolgen. Betont freundlich, wiewohl in einer sprechenden Mischung aus affirmativer Faszination und vorsichtiger Skepsis, schrieb Krueger an Kolbenheyer im Juni 1931:

„Wie ich Ihnen schon in Gera andeutete, habe ich den ermutigenden Eindruck, daß meine und meiner nächsten Schüler wissenschaftliche Ergebnisse sich mit Ihren Einsichten vielfach treffen oder doch berühren. Wenn ich in der Bauhütte [...] streckenweise den Eindruck eines noch etwas schematischen Biologismus hatte, so wird sich das, glaube ich, ausgleichen in dem Maße, in dem die Psychologie biologischer denken lernt und gleichzeitig den Anschluß an die Metaphysik wieder findet. Freilich werden die deutschen Philosophen mehr als bisher von den ordentlich erarbeiteten Befunden der Erfahrungsseelenwissenschaft, auch der experimentellen, Kenntnis nehmen müssen“"20.

„Selbstbesinnung in deutscher Not“: Felix Kruegers Reaktion auf Das Ende Des ERsten WeltKriegs - Auf Wunsch des Senats seiner Universität hielt Krueger am 1. Juni 1919, rund vier Wochen vor der deutschen Unterzeichnung des Versailler Vertrags, im Leipziger Stadttheater eine an die von der Front zurückgekehrten Studenten und Dozenten der Universität Leipzig adressierte Rede mit dem Titel Selbstbesinnung in deutscher Not. In ihr postulierte Krueger, dass mit Kriegsende eine existenziell bedrohliche Ausnahme- und Krisensituation für das deutsche

${ }^{17}$ KAG, Wilhelm Stapel an Erwin Guido Kolbenheyer, 16. Oktober 1929.

${ }^{18} \mathrm{Vgl}$. die Hinweise in Kap. 3.1.2. Krueger war offenbar aufgrund seines regen, völkischem Ideengut verschriebenen volksbildnerischen Engagements als Vortragsredner eingeladen worden.

${ }^{19}$ KAG, Erwin Guido Kolbenheyer an Felix Krueger, 29. Juni 1931 (Durchschlag).

${ }^{20}$ KAG, Felix Krueger an Erwin Guido Kolbenheyer, 23. Juni 1931. 
Volk entstanden sei, die es notwendig, ja alternativlos gemacht habe, alle noch vorhandenen gesellschaftlichen Energien auf die Vertiefung und Vergegenwärtigung „deutscher Art“ ${ }^{\text {“2 }}$ zu konzentrieren. Nach einer einleitenden Verbreitung der Dolchstoßlegende ${ }^{22}$ beschrieb Krueger in seinem Vortrag ein völkisch konnotiertes Gemeinschaftsbewusstsein unter deutschen Frontsoldaten, das er als dezidiert vorbildlich für die gesamte deutsche Nachkriegsgesellschaft verstanden wissen wollte. ${ }^{23}$ Jenes Gemeinschaftsbewusstsein, so Krueger, sei in den Reihen der deutschen Soldaten gegen Kriegsende - im Angesicht der Erkenntnis eines nicht mehr realisierbaren, „sinnlich greifbaren Erfolg[s]“ des deutschen Heeres - noch zusätzlich verstärkt worden; der Glaube „an den unzerstörbaren Kern und Adel unseres Volkes" habe die Frontsoldaten gerade damals mit der Gewissheit der „hohe[n], unverdrängliche[n] Bestimmung des deutschen Volkes“24 erfüllt. Dieser Auffassung versuchte Krueger nicht zuletzt durch angebliche kunsthistorische Erweckungserlebnisse der an der Westfront eingesetzten deutschen Soldaten Plausibilität zu verleihen: „Bei den Vlamen [sic!] bis tief hinein ins nördliche Frankreich, wer hätte nicht mit Inbrunst die Schöpfungen germanischer Kunst betrachtet, mit Wehmut und völkischer Scham die Reste germanischer Sitte und Art?" 25 Im Kern dienten solche Stilisierungen dazu, die Auflösungserscheinungen im deutschen Heer nach der gescheiterten „Michael-Offensive“ im Frühjahr 1918 zu kaschieren ${ }^{26}$, die aufgrund der eklatanten materiellen Unterlegenheit und Mangelversorgung der deutschen Truppen erklärbar und menschlich verständlich waren. Sie passten jedoch nicht in das Bild des „im Felde unbesiegten“ Heeres, wie es bereits unmittelbar nach Kriegsende in der deutschen Öffentlichkeit kolportiert wurde. $^{27}$

Zur Erklärung der Kriegsniederlage rekurrierte Krueger indes nicht nur auf die Dolchstoßlegende. Zugleich behauptete und bemängelte er unzureichende ,geistige Voraussetzungen" für einen deutschen Sieg im Ersten Weltkrieg. Er verwies dabei zunächst auf die in seinen Augen unzureichende Sensibilität der Reichsdeutschen vor 1918 für die Belange und Probleme der „Auslandsdeutschen“. Gerade intellektuelle Eliten („Mitbürger, die zur Führung berufen schienen“) hätten im

${ }^{21}$ Krueger, Selbstbesinnung, S. 16.

22 Krueger verwies auf „Daheimgebliebene“, die „Verweichlichten des Etappendienstes“, die „Drückeberger“ und die „unreifsten unter den Arbeitern“, die „in der Stunde höchster vaterländischer Not" einen „Vernichtungskampf gegen alle Ordnungen des Staates“ eröffnet und sich „weltentrückte[n] Ideologien“ hingegeben hätten. Hierdurch seien die „noch Achtung gebietenden" deutschen Waffen „zerbrochen oder verschleudert" worden. Diese Entwicklung habe einen „entwürdigend[en]" Waffenstillstand nach sich gezogen (ebd., S. 5). Die falschen Grundannahmen und verheerenden gesellschaftlichen Auswirkung der Dolchstoßlegende sind von der Forschung anschaulich gezeigt worden. Vgl. Deist, Zusammenbruch; Barth, Dolchstoßlegende.

${ }^{23}$ Zur Virulenz der Projektion einer pauschalisierten Soldatenerfahrung auf die deutsche und französische Nachkriegsgesellschaft vgl. Kretschmann, Frontgemeinschaft.

${ }^{24}$ Krueger, Selbstbesinnung, S. $6 f$.

25 Ebd., S. 7.

${ }^{26}$ Vgl. Deist, Zusammenbruch; Ders., Militärstreik.

27 Vgl. Sammet, Deutschland, bes. S. 67-71. 
Kaiserreich wissenschaftlichen Arbeiten mit „einem nichtverstehenden Lächeln“ bedacht, die „auf die Erhaltung eigenwertiger Kulturen“ drängten und die „unterdrückte[n] Stammesbrüder"28 jenseits der Reichsgrenzen thematisierten. Die daraus resultierenden Wissensmängel hätten dann im Krieg dazu geführt, dass alle territorialen Annexionsmodelle auf „rein militärischen oder rein wirtschaftlichen Berechnungen" basierten, nicht aber auf einem tieferen Verständnis des deutschen Volkslebens außerhalb der Reichsgrenzen. Zu den ungünstigen geistigen Voraussetzungen für einen deutschen Sieg rechnete Krueger darüber hinaus eine zu große Affinität der „glaubenshungrigen Deutschen“ für die von der gegnerischen Propaganda beschworenen „Menschheitsideale“ - habe diese Leichtgläubigkeit doch dazu geführt, dass die Deutschen noch in ihrem „Unglück und ihre $[\mathrm{r}]$ nationale[n] Schande [...] einen erlösenden, die ganze Menschheit umspannenden Sinn" 29 erkannt hätten.

Indes versuchte Krueger, ähnlich wie Kolbenheyer ${ }^{30}$, aus der deutschen Kriegsniederlage auch positive Folgewirkungen abzuleiten und mit Blick auf die $\mathrm{Zu}$ kunft der Deutschen für größeren Optimismus zu werben. So betrachtete er den Krieg nicht nur als verheerenden Aderlass seines Volks, sondern deutete ihn zugleich - in einer prekären Vermengung psychologischer und sozialdarwinistischer Terminologie - als einen Katalysator der Selektion von Lebenswürdigem und -unwürdigem im deutschen Seelenleben:

„Wertvollstes blieb aufbewahrt in den Besten der Ueberlebenden. Es ist nicht auszusagen, wie viel wir für immer verloren haben: desto mehr müssen wir unsere Kräfte spannen, alle unsere Kräfte, um Neues, Dauerhaftes hervorzubringen. Wer errechnet die Grenzen der Seelenkraft, wer begrenzt die schöpferischen Möglichkeiten eines germanischen Volkes? Die Formen unseres Daseins vor dem Kriege [...] waren nur zum Teil erhaltungswürdig. Der Krieg, dieser erbarmungslose Prüfer, hat alles Unserige ans Licht gezogen, und vieles daran wurde zu leicht befunden. [...] Rüsten wir uns mit jenem langen, geduldigen Willen, der treu sich selbst und ehrfürchtig vor den Ahnen noch den ungeborenen Geschlechtern sich verpflichtet weiß ${ }^{\text {“31. }}$.

Zum Abschluss seiner Rede rückte Krueger dann die Akademikerschaft direkt in den Fokus. Die „Erhaltung des deutschen Geistes“ als wichtigste Voraussetzung zur "Selbsterhaltung" des Volks postulierend, wies er den Universitäten eine zentrale Bedeutung in der Nachkriegsordnung zu. Um ihrer Aufgabe und Verantwortung gerecht zu werden, forderte Krueger die Wissenschaftler dazu auf, ihr im Kaiserreich kultiviertes, bloß inselhaftes Experten- und Spezialistentum zu überwinden und stattdessen nach einer „organischen Einheit des wissenschaftlichen Lebens“ zu streben, ohne dabei jedoch „das Ideal der Gründlichkeit zu opfern“32. Auch sah Krueger die Universitäten vor die Pflicht gestellt, dem „Bildungsstreben der Nichtakademiker" noch „verständnisvoller" und „ausgiebiger" als in der Vergangenheit entgegenzukommen. Dieser „Dienst am Volke“ konnte nach seiner Über-

\footnotetext{
${ }^{28}$ Krueger, Selbstbesinnung, S. 8 (Herv. i. Orig.).

29 Ebd. Krueger gab zudem an, dass es an einer „zureichenden Organisation“ der „geistigen Lebensbedürfnisse" der Fronttruppen gemangelt habe (ebd., S. 8f.).

${ }^{30}$ Vgl. die Hinweise zu Kolbenheyers Broschüre Wem bleibt der Sieg? (1919) in Kap. 2.2.1.

${ }^{31}$ Krueger, Selbstbesinnung, S. 12.

32 Ebd. (Herv. i. Orig.).
} 
zeugung jedoch nur in einem spezifisch „deutsche[n] Sinne“ geleistet werden. In erster Linie sollte das „starke“ und vermeintlich „wissenschaftlich begründete Bewußtsein“ von der „Eigenart“ des deutschen Volks und „dem unverletzlichen Wert“ seiner „schöpferischen Kräfte“33 gepflegt werden. Krueger leitete aus der 1918/19 entstandenen gesellschaftlichen Ausnahmesituation also die Verpflichtung der Universitäten ab, ihre traditionelle „weltweite Offenheit für alles Fremde, das fruchtbar ist“, zurückzustellen und stattdessen den Fokus auf das die spezifische deutsche „Artung“ zu richten:

„Zur Stunde aber gilt es Deutschland herauszureißen aus tiefer Not. Das kann nur durch gesammelte geistige Arbeit gelingen. Alle geistigen Kräfte der Nation müssen gegliedert zusammenwirken zu diesem heiligen Ziel. Ernst, gründliche Selbstbesinnung ist jetzt von jedem einzelnen gefordert. Unsere Rettung kann, heute wie vor hundert Jahren, uns nur erwachsen aus dem sittlich gefestigten, tatbereiten Gefühle für deutsche Art“"34.

Über die öffentliche Wirkung der Rede Kruegers sind wir kaum unterrichtet. Dafür, dass der Vortrag mindestens seitens seiner Kollegen auf positive Resonanz stieß, spricht jedoch ein im Nachlass Kruegers erhaltenes Schreiben des Extraordinarius für Philosophie an der Universität Leipzig Friedrich Lipsius (1873-1934) hin, der im Juli 1919 betonte, Kruegers Worte hätten auch ihm als „Nicht-Kriegsteilnehmer, eine wirkliche innere Erhebung im Leide der Gegenwart“35 bereitet. Ähnlich scheint dies Kruegers Amtsvorgänger Wilhelm Wundt gesehen zu haben: 1939 jedenfalls berichtete Krueger, dass Wundt nach der Lektüre der Rede - sie wurde rasch als Broschüre veröffentlicht - an ihn herangetreten sei und geäußert habe, seine Professur sei offensichtlich „dem rechten Mann“36 übertragen worden.

Zur Vortragstätigkeit Kruegers während der Weimarer Republik - Der Leipziger Rede schloss sich in der Weimarer Republik eine Vielzahl weiterer öffentlicher Vorträge Kruegers an. Eine Auflistung dieser Reden ist in der Festschrift Ganzheit und Struktur überliefert, die 1934 anlässlich des 60. Geburtstags Kruegers erschien. ${ }^{37}$ Die Liste wartet mit einer Vielzahl von Themen auf, die im hier interessierenden Argumentationszusammenhang einschlägig sind. So referierte Krueger bereits im Oktober und November 1918 an den Universitäten Jena und Leipzig zum Thema „Über den deutschen Geist“. Im Publikum saß damals auch der seinerzeit bekannte Leipziger Männerchor-Komponist und Schriftleiter der Deutsche Sängerbundeszeitung Gustav Wohlgemuth (1863-1937), der es sich nach

${ }^{33}$ Ebd., S. 14f. (Herv. i. Orig.).

34 Ebd., S. 15f. (Herv. i. Orig.).

35 UAL, NA Krueger 14, Brief 80: Friedrich Lipsius an Felix Krueger, 4. Juli 1919.

${ }^{36}$ Vgl. Krueger, Klemm, S. 283.

37 Vgl. Klemm/Volkelt/Dürckheim-Montmartin (Hg.), Ganzheit, S. 130-134. In dem vom Universitätsarchiv Leipzig aufbewahrten Nachlass Kruegers finden sich leider nur wenige Aufzeichnungen, die über dessen Vortragstätigkeit nähere Auskunft bieten. Einige handschriftliche Notizen haben sich erhalten, die jedoch kaum zu entziffern sind und zudem oftmals keinen Titel tragen, sodass unklar bleibt, auf welche Veranstaltungen sich die jeweiligen Notizen beziehen. 
Eigenaussage als „deutsch empfindender Mann“ nicht hatte „versagen“ können, der Rede beizuwohnen. Wohlgemuth hob hervor, dass ihn Kruegers Vortrag „wahrhaft aufgerichtet“ habe, und gab seiner Hoffnung Ausdruck, Kruegers Worte mögen „mit Hammerschlägen an die Millionen deutscher Herzen pochen [...], die jetzt in banger Sorge der Zukunft entgegensehen. "38 Neben diesem Vortrag sind noch besonders zu nennen: „Ferienkurse“ der Universität Marburg im August 1922, zu denen Krueger eine Ansprache über „Deutsches Volkstum und Weltanschauung“ beisteuerte; eine Rede über „Deutsche Volkserziehung“ anlässlich der Eröffnung einer Ortsgruppe der Fichte-Gesellschaft in Halle im Dezember 1924; ein Vortrag über „Erziehung zu deutscher Art“ im Oktober 1926 vor Mitgliedern des Neuen Sächsischen Lehrervereins in Chemnitz.

Im Februar 1930 referierte Krueger dann auch vor dem NS-Lehrerbund in Leipzig zum Thema „Geist der Familie und das Volk“. Mit der NS-Bewegung kam Krueger überdies durch eine Mitgliedschaft in Alfred Rosenbergs Kampfbund für deutsche Kultur (KfdK) in Berührung. ${ }^{39}$ Für Mai 1932 ist ein weiterer Vortrag im NSDStB belegt, der Krueger am 23. Mai 1932 für eine „gegen Versailles“ einberufene „Kundgebung in der Wandelhalle der Universität“ einlud. ${ }^{40}$ Vor diesem Hintergrund wird die nach dem jähen Ende seiner akademischen Karriere im März 1938 getätigte Äußerung Kruegers durchaus plausibel, das Leipziger Institut für experimentelle Psychologie sei unter seiner Leitung in den Ruf geraten, eine „völkische Zelle“41 zu sein. Dabei blieben die Vorträge Kruegers freilich nicht ausschließlich auf einschlägige nationalistische Etablissements und Organisationen beschränkt. So hielt er im März und Dezember 1931 zwei Vorträge im Dessauer Bauhaus, zunächst „Über seelische Strukturen“, dann über „Die menschliche Arbeit" ${ }^{\prime 4}$.

Von Interesse ist überdies Kruegers Engagement bei dem vom 15. bis 19. April 1925 veranstalteten „Zweiten Deutschen Akademikertag“ in Elberfeld. Organisiert

38 UAL, NA Krueger 14, Brief 149: Gustav Wohlgemuth an Felix Krueger, 5. November 1918.

39 Vgl. Tilitzki, Universitätsphilosophie, S. 522.

40 Vgl. Krueger, Klemm, S. 287. Die Veranstaltung war nach der Darstellung Kruegers „eine rechte Feierstunde“, zu der er „von besonders kräftig gewachsenen jungen Kommilitonen [...] zwischen zwei Wällen unfreundlich Dreinschauender durchgeleitet“ worden sei.

${ }^{41}$ Krueger, Klemm, S. 289. Der Althistoriker Helmut Berve (1896-1979) hatte diesen Aspekt schon im August 1934 anlässlich Kruegers 60. Geburtstag aufgegriffen. Als damaliger Dekan der Philosophischen Fakultät betonte Berve, ein offizielles Glückwunschschreiben habe - entgegen der sonstigen Gepflogenheiten der Fakultät - im Fall Kruegers seine Berechtigung, da sich Krueger „in den Jahren des nationalen Tiefstandes“ viele Verdienste „zur Pflege des deutschen Volkstums und zur Aufrechterhaltung deutscher Gesinnung“ erworben habe, um „die Wiederauferstehung des deutschen Volkes“ und damit die „nationalsozialistische Revolution“ vorzubereiten. Vgl. UAL, Personalakte Felix Krueger (PA 664), Bl. 64. Zur Person Helmut Berves vgl. Christ, Reichsgedanke.

42 Vgl. Klemm/Volkelt/Dürckheim-Montmartin (Hg.), Ganzheit, S. 131. Bei einem der Vorträge, so erzählte Krueger später, habe der berühmte Luftfahrt-Pionier Hugo Junkers (18591935) erstmalig das Bauhaus betreten, um an die anwesende „international gemischte und politisch radikale Künstlerschar“ zu appellieren, dass Kruegers „Klarstellung der inneren Kräfte" höhere Bedeutung zuzumessen sei als allen technischen Detailfragen ihrer Profession. Vgl. Krueger, Klemm, S. $288 \mathrm{f}$. 
wurde er durch die 1923 gegründete Arbeitsgemeinschaft der völkischen Akademikerverbände des deutschen Sprachgebietes, später umbenannt zu Verband Deutscher Akademiker. ${ }^{43}$ Den ersten „Deutschen Akademikertag“ hatte die Arbeitsgemeinschaft im April 1924 in Potsdam veranstaltet - offenbar nach dem Vorbild der allgemeinen „Deutschen Tage“, wie sie der Deutschvölkische Schutzund Trutzbund seit 1920 zur Sammlung der heterogenen völkischen Szene veranstaltete. ${ }^{44}$ Zum ersten „Deutschen Akademikertag“ war auch Wilhelm Stapel als Referent geladen worden, der zum Thema „Entwicklung des deutschen Volkstums“ vortrug. ${ }^{45}$ Während der Potsdamer Tagung wurden fünf „Grundsätze“ beschlossen, die das ideologische Grundgerüst der Arbeitsgemeinschaft erstmalig absteckten. Demnach sollten das Recht auf staatliche Selbstbestimmung aller Deutschen (einschließlich aller „Auslandsdeutschen“) gestärkt (I) und die „Wehrhaftigkeit aller Deutschen“ sowie der "Schutz des deutschen Volkstums“ soweit „die deutsche Zunge klingt“ (II) gefördert werden. Des Weiteren wollte man jede künftige „politische, wirtschaftliche und kulturelle Arbeit“ auf den „Gemeinschaftsgedanken“ gründen - unter Ablehnung des „Individualismus mit allen seinen Folgerungen“ (III). Die Mitglieder der Arbeitsgemeinschaft erhoben darüber hinaus den Anspruch, „deutsche Führer für unser Volk im Staat, in Wirtschaft, in Kunst- und Schrifttum, vor allem in der Volkserziehung" sein zu wollen (IV), und betonten die Bedeutung der Erziehung der „charaktervolle[n] christliche[n] Persönlichkeit“ durch eine „Erneuerungsbewegung des deutschen Volkes“ (V). ${ }^{46} \mathrm{Als}$ Vorsitzender der Arbeitsgemeinschaft amtierte Karl Hoppmann (1888-1968), einer der „,Chefideologen“ und Vielschreiber“47 aus den Reihen der Deutschen Burschenschaft. Überhaupt waren die „führenden Positionen“ der Arbeitsge-

${ }^{43}$ Die Erforschung dieser Vereinigung ist ein Desiderat. Für Informationen zu ihrer Gründung vgl. Bericht über den Ersten Deutschen Akademiker-Tag. Potsdam 11. bis 14. April 1924, hg. v. Altherrenschaft des Deutschen Hochschulrings, Greifswald 1924. Demnach hatten sich in Karlsbad der Deutsche Akademikerverband Böhmen, der Deutsche Hochschulring und seine Altherrenschaft sowie die Vaterländische Vereinigung Alter Akademiker des Wuppertals, der Verband deutschvölkischer Akademiker für Mähren und Schlesien, der Zentralverband auslanddeutscher Studierender und der Bund Deutscher Hochschüler Rumäniens zusammengeschlossen. Der Beitritt weiterer Verbände wurde unter der Voraussetzung begrüßt, dass sie "nur aus Mitgliedern deutscher Abstammung" bestanden und „volksschädliche Einflüsse“ (S. 5) ausgeschlossen werden konnten.

${ }^{44}$ Bis zum ersten „Deutschen Akademikertag“ in Potsdam waren insgesamt fünf „Deutsche Tage" organisiert worden: In Weimar vom 1.-3. Oktober 1920, in Detmold vom 14.-17. Oktober 1921, in Coburg vom 14.-15. Oktober 1922, in Nürnberg vom 1.-2. September 1923 sowie in Bayreuth am 30. September 1923. Vgl. Lohalm, Radikalismus, passim. Ergänzend: Schramm, Tag.

${ }^{45}$ Neben Stapel sprach der Wiener Ordinarius für Nationalökonomie und Gesellschaftslehre, Othmar Spann (1878-1950), über „Kritik der Demokratie und der wahre Staat“, der Königsberger Theologieprofessor Erich Seeberg (1888-1945) über „Die Bildungshöhe des Akademikers und ihre Pflichten" sowie der Initiator des Berliner Hochschulrings deutscher Art, Otto de la Chevallerie (1894-1972), über „Völkische Akademikerbewegung“.

${ }^{46}$ Vgl. Bericht über den Ersten Deutschen Akademiker-Tag. Potsdam 11. bis 14. April 1924, hg. v. Altherrenschaft des Deutschen Hochschulrings, Greifswald 1924, S. 11.

${ }^{47}$ Ströle-Bühler, Antisemitismus, S. 57. 
meinschaft „von alten Burschenschaftern besetzt ${ }^{“ 48}$. Hoppmann - der 1931 mit dem vor willkürlichen Statistiken berstenden Pamphlet Über den Stand der Verjudung der akademischen Berufe an die Öffentlichkeit trat ${ }^{49}$ - bezeichnete es als das „gemeinsame Ziel aller Einheiten“ seiner Organisation, das „Bewußtsein blutsmäßiger Zusammengehörigkeit unter den deutschen Akademikern der Welt ${ }^{\star 50} \mathrm{zu}$ stärken.

War die Arbeitsgemeinschaft zum Zeitpunkt der Potsdamer Tagung noch eine marginale Randerscheinung des akademischen Lebens gewesen, so stellte sich 1925 beim „Zweiten Deutschen Akademikertag“ in Elberfeld mit rund 6000 Teilnehmern ein bemerkenswerter Erfolg ein. ${ }^{51}$ Auf der fünftägigen Veranstaltung wurden insgesamt neun Ausschüsse gebildet, die sich den Themen „Leibesübungen und Wehrhaftmachung“, „Bildungswesen“, „Kultur und Kunst“, „Rassenfragen“, „Soziale Frage“, „Akademisches Berufsständetum“, „Grenz- und Auslanddeutschtum“ und „Völkische Pressefragen“ widmeten. Hinzu kam ein „Ausschuss für Organisation und Einheitszeitung ".52

Felix Krueger übernahm die Leitung des fünften Ausschusses („Soziale Frage“). Sein Engagement in Elberfeld belegt dabei nicht nur erneut eine grundsätzliche Affinität zur völkischen Bewegung, sondern zugleich Kruegers genuine Überzeugung „, von der Notwendigkeit konkreter sozialpolitischer Reformen “53. In diesem Sinne warnte er in seinem Abschlussbericht über die Ergebnisse seines Ausschusses vor der Vollversammlung des „Akademikertags" vor jedem „Sichabspaltenwollen von der Volksgemeinschaft“. Stattdessen rückte Krueger den „Gedanke[n] des Dienstes" 54 in den Fokus und prononcierte die „völkische Verantwortung" der deutschen Akademiker. Diese sollten aktiver am außeruniversitären Leben Anteil nehmen, um sich auf diesem Weg zu wirklich „sozialen Menschen zu erziehen“. Für besonders bedeutsam hielt es Krueger, auf eine „ständische Durchgliederung des Volksganzen“ hinzuwirken, das „blöde Prinzip der Masse [und] der Mehr-

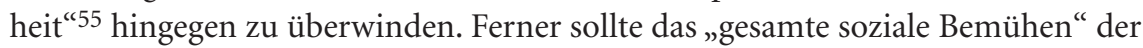
deutschen Akademiker „auf dem Grunde christlicher, völkischer und sozialer Gesinnung erwachsen“; sie hätten sich „einzuordnen in den Gemeinschaftsdienst am großdeutschen Volkstum“"56. In seinen Ausführungen knüpfte Krueger zu-

${ }^{48}$ Leisen, Ausbreitung, S. 110.

${ }^{49}$ Die Schrift wurde 1934 in der vom Rassenpolitischen Amt der NSDAP herausgegebenen Broschüre Warum Arierparagraph? als sogenanntes Beweismaterial herangezogen. Vgl. Schulz/ Frercks, Arierparagraph, S. 42. Zum Prozentsatz jüdischer Studierender in Relation zum Anteil an der Gesamtbevölkerung: Titze, Hochschulstudium, S. $226 f$.

${ }^{50}$ Hoppmann, Verband, S. 572.

${ }^{51}$ Vgl. Volk in Not! Ein Weck- und Mahnruf der Arbeitsgemeinschaft der Völkischen Akademiker-Verbände des Deutschen Sprachgebietes, o. O. ${ }^{2} 1925$, S. 7.

$52 \mathrm{Zu}$ den ausführlichen Berichten über die Ergebnisse der jeweiligen Ausschüsse vgl. ebd., S. 17-67. Als "Einheitszeitung" lief in der Folgezeit die aus der Deutschen Hochschulzeitung hervorgegangene Deutsche Akademiker-Zeitung (1925-1941).

${ }^{53}$ Tilitzki, Universitätsphilosophie, S. 529.

54 Volk in Not!, S. 36 f.

55 Ebd., S. 38.

${ }^{56}$ Ebd., S. $41 \mathrm{f}$. 
gleich an Denkmuster der Eugenik an: „Krüppel“ und „Idioten“ schloss er ausdrücklich aus der „Wohlfahrt des Volksganzen“ aus, auf die er die deutschen Akademiker verpflichten wollte. ${ }^{57}$ Durch eine für das Jahr 1935 belegte Äußerung wird diese Neigung bestätigt ${ }^{58}$, näher schriftlich ausgeführt hat Krueger diese Thematik jedoch nie.

Kruegers Haltung IM „Dritten Reich“ - Kruegers Denken stand nach 1933 in einem Spannungsverhältnis zwischen der grundsätzlichen Befürwortung der neuen politischen Ordnung und der genuinen Sorge um die Erhaltung und Pflege wissenschaftlicher Standards an den deutschen Universitäten. Anschaulich geht dies aus der Antrittsrede Kruegers bei der Übernahme des Rektorenpostens der Universität Leipzig im April 1935 hervor, die er dem Thema „Seelisches Sein und nationale Formwerdung“ widmete. Einem Bericht der Leipziger Tageszeitung vom 9. April 1935 zufolge bestand Kruegers „erste Handlung“ bei der Antrittsrede darin, einen „Heilruf auf das Volk und den Führer“ anzubringen. Den Inhalt der Rede fasste das Blatt wie folgt zusammen:

„Es war eine weitgespannte und jeden Anteilnehmenden innerlich aufschließende Lebensschau aus dem Schaffensgeiste des weithin wirkenden fruchtbaren Leipziger psychologischen Forschungskreises - mit wahrhaft verpflichtender Zielrichtung auf die Vervollkommnung unseres seelischen und volklichen Daseins und Werdens. [...] Die bereichernde Rede gipfelte in den Erkenntnissen, daß wir alle als von wahrem Werde-Willen Erfüllte einen wesenhaften Zusammenhang aller Erscheinungen und Erlebnisse in einem Überdauernden, unendlich Ganzen erstreben und finden müssten und daß Volkstum und Reich die im vergehenden irdischen Sein am zuverlässigsten bestehenden sinnbildlichen Hochgestalten des Ewigen sind“59

Krueger begnügte sich jedoch nicht mit einem reinen Loblied auf den Nationalsozialismus. Er forderte zugleich die Rückkehr zur universitären Normalität ein, wie einem Artikel der Leipziger Neuesten Nachrichten zu entnehmen ist, der ebenfalls am 9. April 1935 erschien. Krueger hob demnach im Besonderen die „Notwendigkeit“ hervor, dass die „studentische Jugend“ nach ihrem „vorbildlichen politischen Einsatz“ während der nationalsozialistischen Revolution wieder zu „wissenschaftlicher Leistung“ zurückfinden müsse; in keinem Fall dürfe die Tradition der „deutschen Weltgeltung" 60 in der Wissenschaft einem banalen, unreflektierten Anti-Intellektualismus geopfert werden. Auch um eine Trivialisierung seiner eigenen Forschungen war Krueger besorgt, insbesondere seiner „Ganzheitspsychologie“. So warnte er 1935 mit Blick auf die weitere Entwicklung der

57 Ebd., S. 39f.

58 „Des Staates Wehr und Gerichtsbarkeit können der Härte nicht entbehren. Herrisch fordert er Opfer des Eigenwillens und sogar des Lebens, wie sich allerwegen einem Ganzen, das Bestand haben will, noch seine edelsten Teile zu fügen haben. Den Menschen ist gegeben, daß sie erkennen können, was an ihrem Dasein unganz, das heißt lebenswidrig und formungsfeindlich ist. Sie bringen das Opfer ihrer Fehlsamkeit, indem sie mit Bewußtsein ihrem Staat gehorchen und freiwillig geordnete Gewalt über sich anerkennen“. Zitiert nach: Harrington, Suche, S. 332.

${ }^{59}$ Leipziger Tageszeitung [für nationalsozialistische Politik, Kultur und Wirtschaft], 9. April 1935. Zitiert nach: UAL, Personalakten PA 664, Bl. 69 (Herv. i. Orig.).

${ }^{60}$ Leipziger Neueste Nachrichten, 9. April 1935. Zitiert nach: UAL, Personalakten PA 664, Bl. 70. 
Psychologie als Wissenschaft, dass die ganze „Familie [...] der schwierig zu deutenden Wörter“, die „mit ,ganz' zusammenhängen“, infolge einer unreflektierten Verwendung des Begriffs „Volksgemeinschaft“ Gefahr laufe, ins bloß „Gemeinplätzliche“ zu „entart[en]“61.

Parallel dazu kam Krueger jedoch den hochschulpolitischen und rassetheoretischen Vorstellungen der Nationalsozialisten entgegen, indem er betonte, dass jedes „Streben nach Wahrheit immer rassische und völkische Wurzeln“62 besitzen müsse. Diese Anlehnung an eine rassenbiologisch konnotierte Erkenntnistheorie erinnert an Prämissen aus Kolbenheyers Philosophie der Bauhütte ${ }^{63}$ und war im Falle Kruegers mehr als ein bloßes Lippenbekenntnis: Wohl blieb „Rasse“ für Krueger ein in erster Linie psychologischer, „primär als Geist, Seele oder ,Gestalt “"64 verstandener Begriff - seine Vorbehalte gegen ein monokausal biologistisches Denken ohne geisteswissenschaftliche Korrektive legte der Psychologe keineswegs ab. Gleichwohl forderte er 1935 in seiner Funktion als Präsident der Deutschen Gesellschaft für Psychologie sein Fachpublikum unmissverständlich zu einer stärkeren Integration eines erbbiologisch verstandenen Rassebegriffs als analytische Kategorie auf.

„Dabei wird es bleiben: Wo immer wir in der Erfahrung Menschliches vorfinden, erweist es sich als mitbedingt durch die übrige ,Natur', insonderheit als erdgebunden und zu allermeist als leibverhaftet; [...] Von den Zusammenhängen des Menschen mit der Natur werden jetzt aus guten Gründen jene Erbfaktoren auf das stärkste berücksichtigt, die - allzu lange vernachlässigt - in dem Begriff, Rasse' einen greifbaren Kern ausmachen" ${ }^{\prime 65}$.

Vereinzelt argumentierte Krueger freilich schon vor 1933 mit der Kategorie „Rasse“. In einem Artikel der Blätter für deutsche Philosophie aus dem Jahr 1932 warnte er etwa davor, dass das „Abendland [...] dem Chaos anheimfallen“ und „minder edle Rassen die Oberhand gewinnen“ würden, falls nicht einer seinem ganzheitlichen Denken entsprechenden „Reformation die Bahn frei“ gemacht werde. ${ }^{66}$ Nach der NS-„,Machtergreifung“ wollte Krueger daher auch „keinen Zweifel“ aufkommen lassen, dass „die praktische Rassenpflege auf dem rechten Wege“ sei, wenn sie „mit allen zweckdienlichen Mitteln“ danach strebe, die „als ,nordisch bestimmbaren“ Vererbungsmerkmale zu „verstärken“, hätten sich diese doch „als besonders lebenstüchtig und für das Kulturschaffen als am meisten förderlich“67

${ }^{61}$ Krueger, Psychologie, S. 27. Bei diesem Text handelte es sich um eine erheblich erweiterte Fassung der Ausführungen Kruegers auf dem 14. Kongress der Deutschen Gesellschaft für Psychologie, der im Mai 1934 in Tübingen stattgefunden hatte.

62 Leipziger Neueste Nachrichten, 9. April 1935 (zitiert nach: UAL, Personalakten PA 664, Bl. 70).

${ }^{63}$ Vgl. Kap. 3.3.1.

64 Breuer, Völkischen, S. 9.

${ }^{65}$ Krueger, Psychologie, S. 13.

66 Ders., Problem, S. $138 \mathrm{f}$.

67 Ders., Psychologie, S. 13f. Die Vorstellung, die „nordische“ Rasse besitze vor allen anderen Rassen besonders kulturschöpferische Anlagen, war ein von der völkischen Bewegung des wilhelminischen Kaiserreichs popularisiertes Ideologem, das während der Weimarer Republik insbesondere durch die Schriften Hans F. K. Günthers einem breiteren Publikum vermittelt wurde. Aus seinen „rassenkundlichen“ Studien hatte Günther seit den frühen 1920er Jah- 
erwiesen. Die Rassenforschung sollte jedoch künftig andere Faktoren und Perspektiven noch stärker mitberücksichtigen und einkalkulieren, insbesondere das „durch ein Gemeinschaftsleben bedingt[e]“ und historisch gewachsene „Seelentum “68 der Deutschen. Um die „Wissenschaft der menschlichen Seele“, als deren führender Vertreter er sich verstand, gegenüber der Biologie nicht gänzlich ins Hintertreffen geraten zu lassen, stellte Krueger heraus, dass die „Erbmasse eines Volkes“ zwar „dem Zufall oder willkürlichen Eingriffen entzogen“ sei, jedoch stets „von psychischen Anlagen durchsetzt“69 werde. Zugleich sprach er sich dagegen aus, „Lebenseinheiten von rassisch geprägter Form“, die von der eigenen abwichen, allein aufgrund dieses Abweichens anzufeinden. Unter den fremden Formen, so Krueger, gebe es „gewiß viele“, die „erhaltungswürdig“ seien. Die Existenz nicht erhaltungswürdiger „rassischer Lebenseinheiten“ ließ diese Äußerung allerdings ausdrücklich offen, mit allen damit verbundenen prekären Implikationen.

\title{
4.2 Andreas Thomsen und „Der Völker Vergehen und Werden"
}

\begin{abstract}
Gibt es für einen weitschauenden Politiker eine schönere und zugleich wichtigere Aufgabe, als die Natur daran zu hindern, die von ihr geschaffenen Edelvölker wieder untergehen $z u$ lassen, selber aber gleichzeitig neue heranzuzüchten, die langlebiger und vielleicht auch glücklicher und beglückender sind als die von der Natur geschaffenen, wildgewachsenen?70
\end{abstract}

Zum Werdegang Thomsens und seiner Bezienung zu Hans Grimm - Man mag sich an Thilo Sarrazin erinnert fühlen. Ebenso wie vor 2010 kaum jemand von dem langjährigen Mitarbeiter des Bundesfinanzministeriums und Ex-Vorstandsmitglied der Deutschen Bundesbank mit der Veröffentlichung des Pamphlets Deutschland schafft sich $a b^{71}$ rechnete, dürfte sich auch im Jahr 1925 mancher verwundert die Augen gerieben haben, als Andreas Thomsen (1863-1948) mit der Broschüre Der Völker Vergehen und Werden. Grundlage einer allgemeinen Völkerpolitik an die Öffentlichkeit trat. ${ }^{72}$ Dem eigentlichen Fachgebiet des damals kurz

ren ein „unvergleichlich“ häufigeres „Auftreten schöpferischer“ und „hervorragender“ Menschen in der „nordischen Rasse“ postuliert und unter anderem daraus spezifische Vorrechte abgeleitet. Günther hing dabei jedoch nicht dem Glauben an, dass sich die deutsche Bevölkerung im Lauf ihrer Geschichte rein oder auch nur vorwiegend "nordisch“ erhalten habe. Stattdessen schätzte er den Anteil „rein nordisch“ verbliebener Menschen an der deutschen Bevölkerung lediglich auf 6-8\%. Vgl. Günther, Rassenkunde, S. 152, 208.

${ }^{68}$ Krueger, Psychologie, S. 14 (Herv. i. Orig.).

${ }^{69}$ Ebd., S. 13.

${ }^{70}$ Leitsatz in: Thomsen, Völker.

${ }^{71}$ Hierzu: Hentges, Racism; Werner, Sarrazin.

72 Bestenfalls Insider konnten dies kommen sehen. Zwar hatte Thomsen 1922 in einer kurzen Broschüre bereits Überlegungen zum Aussterben der modernen Kulturvölker angestellt, diese 
vor der Emeritierung stehenden Strafrechtsprofessors lag diese Thematik jedenfalls denkbar fern. Thomsen, am 1. Juni 1863 in Hannover geboren, hatte an den Universitäten Freiburg, Göttingen, Leipzig und Lausanne Rechtswissenschaften studiert. Seiner Promotion an der Universität Göttingen im Jahr 1886 schloss sich ein Studium der Mathematik und Physik an, ehe Thomsen sich 1893 schließlich an der Universität Kiel für Strafrecht habilitierte. Anschließend war er in Kiel als Privatdozent tätig. Im Oktober 1902 wurde Thomsen zum Extraordinarius für Strafrecht, Strafprozeß-, Zivilprozeß- und Kirchenrecht in Münster berufen. ${ }^{73}$

Während des Ersten Weltkriegs leistete Thomsen freiwillige Kriegshilfe und unterzeichnete im Oktober 1914 die Erklärung der Hochschullehrer des Deutschen Reiches. Ihr zufolge war das „Heil“ der „ganze[n] Kultur Europas“ von dem „Siege“ abhängig, „den der deutsche ,Militarismus“ erkämpfen“ würde, „die Manneszucht, die Treue, der Opfermut des einträchtigen freien Volkes“74. Im Oktober 1917 gehörte Thomsen zudem zu den Trägern jener „Vaterländischen Kundgebung“ gegen die Friedensresolution des Deutschen Reichstags vom 19. Juli 1917, aus der die Deutsche Vaterlandspartei hervorging. Ob Thomsen zugleich Mitglied dieser Partei wurde, ist ungewiss. ${ }^{75} 1919$ wurde Thomsens Stelle an der Universität Münster „in ein etatsmäßiges Extraordinariat umgewandelt“76. Ein Antrag „auf Ernennung zum persönlichen Ordinarius“ blieb hingegen erfolglos, da dem Ministerium Thomsens „bisherige wissenschaftlichen Leistungen“ $\mathrm{zu}$ diesem Schritt „nicht ausgereicht“77 hatten. Im September 1928 wurde Thomsen emeritiert, führte seine Lehrtätigkeit an der Universität Münster jedoch für acht weitere Jahre fort. ${ }^{78}$ Nähere Informationen zum Lebensweg Thomsens im „Dritten Reich“ ließen sich nicht erheben. Es darf jedoch vermutet werden, dass der in seinem achten Lebensjahrzehnt stehende Emeritus öffentlich kaum mehr in Erscheinung trat. Nach seinem Tod im Frühjahr 1948 wurde Thomsen in der Entscheidung um die Pensionsbezüge seiner Witwe posthum für „völlig unbelastet“ erklärt. ${ }^{79}$

Wie aber kam der kurzfristige Kontakt zwischen dem Strafrechtslehrer und Hans Grimm im Winter 1928/29 zustande? Die Initiative ging von Thomsen aus. Wie bei so vielen anderen war auch für seine Entscheidung, den Kontakt mit dem Dichter zu suchen, die Lektüre von Volk ohne Raum ausschlaggebend gewesen;

Schrift ist heute in den deutschen Bibliothekskatalogen jedoch eine wahre Rarität. Daher ist hier von einer minimalen Resonanz auszugehen. Außerdem war Thomsen als Vorsitzender des westfälischen Provinzialverbands des 1922 gegründeten Reichsbundes der Kinderreichen Deutschlands (vgl. Thomsen, Völker, S. 42. Ebd. auch die von Thomsen verfassten Leitsätze des Bundes) hervorgetreten, dessen rassehygienischer Einschlag bereits in der Forschung skizziert worden ist. Vgl. Stephenson, Reichsbund.

${ }^{73}$ Vgl. Steveling, Juristen, S. 122. Ebd. auch die Vermutung Stevelings, dass Andreas Thomsen "(vermutlich) der Sohn des L[and]G[ericht]-Direktors Thomsen“" war.

${ }^{74}$ Vgl. den Abdruck der Erklärung in Böhme (Hg.), Aufrufe, S. $49 \mathrm{f}$.

${ }^{75}$ Vgl. Steveling, Juristen, S. 153, 159, 161.

76 Dies., Geschichte, S. 534.

77 Dies., Juristen, S. 278, Anm. 130.

78 Vgl. ebd., S. 261.

79 Ebd., S. 576. 
offenbar hatte der frisch emeritierte Strafrechtler nun endlich Zeit für die umfangreiche Lektüre gefunden. Da Thomsen in dem Autor des Romans nicht ohne Grund eine weltanschaulich verwandte Seele erkannte, fügte er seinem ersten Brief an Grimm vom 27. Dezember 1928 mehrere „Heftchen“ bei, unter anderem zu den Themen Die deutschen Familienverbände als Völkerkeime, als Retter des schwindenden deutschen Volkes und Die Bildung von Völkerkeimen zur Erhaltung und Mehrung wertvoller Erbanlagen. ${ }^{80}$

Mit dem Komplex „Völkerkeime“ griff Thomsen das Kernelement seiner 1925 in Leipzig erschienenen Broschüre Der Völker Vergehen und Werden heraus. Die sich aufdrängende Frage, wie er als Jurist dazu gekommen war, sich mit dieser so fachfremden Thematik auseinanderzusetzen, beantwortete Thomsen in dem Vorwort seiner Schrift mit dem Verweis auf die illuminierende Lektüre mehrerer „biologische[r] Schriften“. Um welche Arbeiten es sich dabei gehandelt hat, lässt sich über das knappe Literaturverzeichnis der Broschüre unschwer rekonstruieren. Die Liste liest sich wie das „Who is Who“ des deutschsprachigen Eugenikund Rassenhygiene-Diskurses der ersten Hälfte des 20. Jahrhunderts ${ }^{81}$ : Neben Wilhelm Schallmayers Hauptwerk Vererbung und Auslese im Lebenslauf der Völker $\left({ }^{4} 1920\right)$ stützte sich Thomsen auf Hans F. K. Günthers Rassenkunde des Deutschen Volkes ( $\left.{ }^{3} 1923\right)$, auf Walter Scheidts Einführung in die naturwissenschaftliche Familienkunde (1923) sowie auf den von Erwin Bauer, Eugen Fischer und Fritz Lenz herausgegebenen, einflussreichen Grundriß der menschlichen Erblichkeitslehre und Rassenhygiene (1921). ${ }^{82}$ Erst durch diese Lektüre, so Thomsen, sei ihm klar geworden, dass „ganze Verbrechervölker“ im Entstehen waren, die für die „menschliche Gesellschaft eine ständige Plage und Gefahr bilden und in ihrer Krankhaftigkeit und Minderwertigkeit sich schließlich selbst zur Last“ fallen würden. Als Jurist und „Kriminalpolitiker“ sei es eigentlich seine Aufgabe gewesen, „die Entstehung solcher Verbrechervölker zu verhindern“, doch habe er schließlich die Entscheidung getroffen, sich „zunächst mit dem Wichtigeren, mit der ,Erhaltung der verschwindenden heutigen und Heranzüchtung neuer Kulturvölker` $[\ldots] \mathrm{zu}$ beschäftigen" 83 .

Die INNERE UND ÄUSSERE BEDROHUNG DER „EDELVÖLKER“ - Thomsen untergliederte seine Schrift in insgesamt zehn Kapitel, von denen die ersten fünf einen Abriss der globalen Bevölkerungsentwicklung in Vergangenheit, Gegenwart und Zukunft bieten. Die Darstellung ist maßgeblich von der These durchdrungen, dass Gebur-

${ }^{80}$ Vgl. DLA, A:Grimm, Andreas Thomsen an Hans Grimm, 27. Dezember 1928.

${ }^{81}$ Die Literatur zu den Themenkomplexen Eugenik und „Rassenhygiene“ seit dem wilhelminischen Kaiserreich bis ins „Dritte Reich“ ist gerade auch durch verdienstvolle regionale und lokale Spezialstudien bei Weitem zu ausdifferenziert, als dass an dieser Stelle eine erschöpfende Bibliografie gegeben werden könnte. Einführend zu diesem Themenkomplex: Weingart/ Kroll/Bayertz, Rasse.

82 Als weitere Literatur ist zudem Ursachen und Bekämpfung des Geburtenrückganges im Deutschen Reich (1914) von dem österreichischen Bakteriologen und von 1902 bis 1923 Ordinarius für Hygiene an der Universität München Max von Gruber (1853-1927) aufgeführt.

83 Thomsen, Völker, S. X. 
tenrückgang ${ }^{84}$ und „Rassenvermischung“ als die beiden historisch verheerendsten „Völkermörder“ betrachtet werden müssten. Durch sie werde der Restbestand der modernen „Kulturvölker" bedroht und gerate in einen schleichenden Prozess des Absterbens. ${ }^{85}$ Den exklusiven Ehrentitel „Kulturvolk“ sprach Thomsen dabei neben Deutschland und England nur Frankreich und - mit erheblichen Abstrichen - den USA ${ }^{86}$ zu. In den restlichen Kapiteln diskutierte Thomsen dann mögliche, nach seiner Ansicht notwendige Maßnahmen zur dauerhaften Erhaltung der „Kulturvölker“, wobei er in erster Linie für die Bildung „Biologischer Völkerkeime“ plädierte, deren Gestalt weiter unten erläutert wird. In der völkischen Szene lag dieses Thema damals in der Luft, erinnert sei nur an den Gründer des Deutschbunds Max Robert Gerstenhauer und seine Forderung nach einer „Bildung germanischer Rassenkerne ${ }^{\text {“87 }}$.

Worin aber bestanden für Thomsen die Ursachen des „Absterbens der Kulturvölker“? Als ein Hauptfaktor galt ihm die zu geringe Reproduktionsrate „der oberen Stände“, verstanden als soziale und geistige, aber auch biologische Elite. Als das absolute Mindestmaß für die Überlebensfähigkeit eines Volks gab Thomsen die Zahl von vier Kindern pro Ehe aus - eine Quote, die beispielsweise von der höheren Beamtenschaft seit langer Zeit nicht annähernd eingelöst worden sei. Auch die deutschen „Geistesarbeiter“ nahm Thomsen in die Pflicht, indem er ihre geringe Kinderfreude rügte, deren Ursachen er auf „körperliche Unfähigkeit“ oder aber den egoistischen Hang „zu gesellschaftlichen Äußerlichkeiten, Geldgier und Trägheit“ 88 zurückführte. Das daraus resultierende „Absterben der Völker von oben herab" 89 ging in den Augen Thomsens mit einem kontinuierlich wachsenden Übergewicht der (ungleich zeugungsfreudigeren) „unteren Stände“ einher - eine schleichende "Verpöbelung" und „Entedelung der Völker“. Sollte es, so Thomsens Fazit, nicht gelingen, die Nachkommenzahl der „führenden Menschen und [...] führenden Familien" signifikant zu steigern, würden früher oder später „alle guten Elemente des deutschen Volkes und mit ihnen Kunst und Wissen-

$84 \mathrm{Zu}$ dem im ersten Drittel des 20. Jahrhunderts äußerst intensiv und kontrovers debattierten Thema des Geburtenrückgangs vgl. Ferdinand, Geburtenrückgangstheorien; Matz, Bewertung.

85 Thomsen, Völker, S. 11. Historisch relevante „Völkermörder“ wie Naturkatastrophen, Hungersnöte, Epidemien und Krieg konnten laut Thomsen demgegenüber in der Moderne beim Verschwinden ganzer Völker keine entscheidende Rolle mehr spielen.

${ }^{86}$ Den „Yankees“ als der angeblich hochwertigsten Bevölkerungsgruppe der USA diagnostizierte Thomsen, sie würden aufgrund ihrer zu geringen Reproduktion im Vergleich zur schwarzen Bevölkerung bereits „in 2-3 Generationen ausgestorben sein“. Anschließend werde „das zweittüchtigste der Völker" der USA „die oberen Stände ausfüllen“, nur um selbst alsbald aus dem gleichen Grund wie die „Yankees“ auszusterben. Übrig blieben am Ende „schließlich die Mulatten und zu allerletzt die Nachkommen der 10 Millionen Neger [...] Sklaven glaubten die Yankees einzuführen, und Erben haben sie eingeführt!" (ebd., S. 20, Herv. i. Orig.).

${ }^{87}$ Vgl. Breuer, Völkischen, S. 165; Deutschbund-Blätter 32 (1927), Nr. 8/9, S. $47-49$ (für den Hinweis auf diese Quelle danke ich Alexandra Esche).

88 Thomsen, Völker, S.18f.

${ }^{89}$ Ebd., S. 19. 
schaft und alles Schöne und Edle [...] verschwinde[n]“. Am Ende bleibe „nur noch die Hefe übrig"90.

Mit dieser Argumentation reproduzierte Thomsen Vorwürfe, die sich in praktisch identischer Form schon in den Debatten um die "Soziale Frage“ während des 19. Jahrhunderts finden. Als prominentes Beispiel sei auf den Philosophen Eduard von Hartmann verwiesen, der in seinem Werk Moderne Probleme (1886) vehement vor den Folgen einer unzureichenden Reproduktionsrate der „besseren" Gesellschaftsschichten gewarnt ${ }^{91}$ und sogar elf Kinder als das biologische Normalmaß ausgegeben hatte. ${ }^{92}$ Neu bei Thomsen war jedoch, dass er die Gefahr des „Absterbens“ der „Kulturvölker“ durch einen externen Faktor dramatisch zugespitzt sah: den enormen Populationsdruck des chinesischen Volks. Diese Diagnose überrascht insofern, als die chinesische Bevölkerung zwischen 1800 und 1913 zwar von rund 300 auf rund 440 Millionen anwuchs, die Wachstumsrate des Landes jedoch deutlich hinter jener Westeuropas, Russland, Lateinamerikas, der USA und auch Japans zurückgeblieben und in dem Zeitfenster 1820-1870 sogar negativ ausgefallen war. ${ }^{93}$ Dessen ungeachtet klassifizierte Thomsen die Chinesen als „die arbeitsamste, zäheste und bedürfnisloseste Rasse“ der Erde, der „selbst nicht das jüdische“ Volk „im Kampfe ums Dasein gewachsen“94 sei. In einer demografischen Variante der schon im Kaiserreich intensiv diskutierten „Gelben Gefahr"95 skizzierte Thomsen ein dystopisches Szenario, in der das chinesische Volk bereits im Jahr „2200 die ganze Menschheit“, also die gesamte Weltpopulation, stellen würde. Diese Überlegung basierte auf der Annahme, dass China auf lange Sicht das einzige Volk bleiben werde, das einen Geburtenrückgang verhindern und sich darüber hinaus durch abschottende Heiratsregelungen besonders effektiv vor einer rassischen „Vermischung“ schützen werde.

Den restlichen Völkern prophezeite Thomsen hingegen, sich sukzessive zu einem „einzigen großen Weltmischvolk“ vermengen zu werden. Als Ursachen gab er zum einen fehlende Sensibilität für die Bedeutung von Rassereinheit an, zum anderen verwies Thomsen auf die ungeahnte Mobilität verleihenden modernen Verkehrsmittel. Jenes „Weltmischvolk“ werde sich rasch „entedeln und infolge Geburtenrückgangs an Zahl fortwährend abnehmen"96 und den Chinesen da-

90 Ebd., S. 22f. (Herv. i. Orig.).

91 Vgl. Hartmann, Probleme, S. 50-73.

92 Konkret belief sich die „naturgemässe Kinderzahl einer nationalen Ehe von jugendlich verbundenen gesunden und kräftigen Gatten“ laut Hartmann auf „2 vor dem 20. Jahre, 5 in den 20ger, 3 in den 30ger und 1 in den 40ger Jahren“ (ebd., S. 57).

93 Osterhammel, Verwandlung, S. 184-192. Erst in der zweiten Hälfte des 20. Jahrhunderts stieg die Bevölkerung Chinas - parallel zu jener Indiens - extrem an.

94 Thomsen, Völker, S. 32.

95 Eine Zusammenfassung der politisch-militärischen, wirtschaftlichen und sozialen Dimension der Debatte um die „Gelbe Gefahr“ im ausgehenden Deutschen Kaiserreich bietet: Mehnert, Deutschland, S. 35-59. Der Umgang mit China als literarischem Thema ist gut erforscht durch die Habilitationsschrift: Liu, Exklusion. Zum Aspekt der „Gelben Gefahr“ hier bes. S. 116-132.

96 Thomsen, Völker, S. 28. 
durch immer größere Freiräume zur Ausbreitung bieten. Vor dem endgültigen demografischen Triumph der Chinesen erwartete Thomsen jedoch zunächst einen Einbruch der slawischen Völker „im ganzen germanisch-romanischen Westeuropa" ${ }^{\text {"97 }}$, da diese im Vergleich mit den mittel- und westeuropäischen Völkern einen deutlich langsameren Geburtenrückgang aufwiesen. Die „Verslawung“ des Abendlandes werde jedoch nur eine Etappe bleiben, da sich auch ein slawischeuropäisches „Mischvolk“ der Chinesen nicht werde erwehren können. Ein Aufgehen der Chinesen in jenem „Mischvolk“ hielt Thomsen für unwahrscheinlich. ${ }^{98}$

Dem - aus völkischer Perspektive - historischen „Dilemma der im Laufe der Jahrhunderte durch Kontakte mit anderen Völkern“ unweigerlich „verlorengegangenen Rassereinheit ${ }^{\text {"99 }}$ war sich dabei auch Thomsen bewusst. Dass sich die Rassen bis zur Gegenwart vollkommen rein erhalten hätten, verwarf er als naives Wunschdenken 100 - eine Deutung, die er Hans F. K. Günthers Rassenkunde des Deutschen Volkes entnommen haben dürfte. ${ }^{101}$ Trotz dieser Relativierung gab Thomsen, ebenso wie Günther, das Ideal der Reinrassigkeit jedoch nicht grundsätzlich auf. Vielmehr weinte er ihrem Verlust heiße Tränen nach, wie aus seinen Ausführungen über die antiken „Athener“ hervorgeht. Dass es diesen nicht gelungen sei, sich in ihrer ursprünglichen rassischen Konstitution zu erhalten, galt Thomsen als tragisch. Von welchen Leistungen in „Kunst und Wissenschaft", so rief er aus, hätte die Menschheit profitiert, hätten sich „sagen wir einmal 2030000 reine Nachkommen von Athenern“ erhalten; was wäre für die „Zukunft noch zu erwarten“ gewesen, gäbe es "heute noch 15 Millionen reinrassiger Griechen“. Stattdessen, so Thomsens gehässige Randnotiz, müsse man sich nun mit „ca. 15 Millionen einigermaßen rassereinen Juden“ 102 herumschlagen. Wie stark Thomsen am Ideal der Rassereinheit festhielt, ist darüber hinaus daran ablesbar, dass er die eherne Notwendigkeit beschwor, zumindest den noch vor-

97 Ebd., S. 27.

98 Vgl. ebd., S. 31 f.: „Das chinesische Volk wird demnach - es sei denn, daß etwas Außerordentliches geschieht - seinen schon seit vielen Jahrhunderten dauernden Siegeslauf in der Weise fortsetzen, daß es sich nach allen Seiten hin ausdehnt. [...] Die Völker, die es vorfindet, treibt es vor sich her oder, richtiger gesagt, sie weichen vor ihm zurück, denn in die kompakten Massen der Chinesen werden sie nicht hineingehen, weil sie dort wirtschaftlich erst recht nicht existieren können. Die Chinesen werden daher überall gleichsam in menschenleeres Gebiet einwandern. [...] Eine Mischung wird voraussichtlich nicht stattfinden, wenigstens kommen heut Mischehen zwischen Chinesen und Nichtchinesen so gut wie gar nicht vor".

99 Puschner, Germanenideologie, S. 89.

100 Vgl. Thomsen, Völker, S. 11: „In alter und schon in vorhistorischer Zeit sind die Völker der Erde derartig durcheinandergewandert und so zusammengewürfelt worden, daß wir nicht ein einziges rassereines Volk mehr besitzen“.

101 Auch Günther hielt „Reinrassigkeit“ jedenfalls für ein sehr rares Phänomen: „Die gegenseitige Zerkreuzung [sic!] aller europäischen Rassen ist so stark vorgeschritten, daß reinrassige Menschen äußerst selten sind. [...] Menschen, deren Körper alle Merkmale einer und nur dieser einen Rasse zeigt, sind ziemlich selten. Man schätzt zu hoch, wenn man annimmt, daß etwa ein Zehntel aller Deutschen noch rein nordisch sei. Die rein nordischen Deutschen mögen eher 6-8\% des deutschen Volkes ausmachen" (Günther, Rassenkunde, S. 208).

102 Thomsen, Völker, S. 34. 
handenen Reinheitsgrad zu konservieren, auch wenn dieser nicht mehr genau zu bestimmen sei. Welche Vorschläge aber machte Thomsen, um dieses Ziel zu erreichen?

Agenda 2200: Bildung und BesChaffenheit DER „Biologischen VÖLKerKeIME“ Dieselbe Trauer, die Thomsen im Jahr 1925 über das Aussterben der kulturell und geistig elitären „Athener“ empfand, prophezeite der Strafrechtslehrer dem chinesischen Volk im „Jahre 2200“, sollten sich bis dahin keine „möglichst reine[n] Nachkommen der heutigen Kulturvölker" erhalten. Restbestände des deutschen, englischen und französischen Volks, etwa in der Stärke von „100 Millionen“, brächten dereinst den „etwa 2400 Millionen“ Chinesen „viele Kulturgüter“, die diese „mit ihren spezifisch chinesischen Begabungen“ aus eigener Kraft niemals „hervorbringen könnten" ${ }^{103}$. Vor diesem Hintergrund forderte Thomsen, zügig und zielstrebig möglichst reinrassige Enklaven zu bilden, die auch unter einer künftigen chinesischen Übermacht, die Thomsen als unaufhaltsam galt ${ }^{104}$, überlebensfähig sein würden. Konkret galt es „Gemeinden“ zu gründen, welche die europäischen Kulturvölker "jeweils im Kleinen“105 repräsentieren, sich einer weiteren rassischen Vermischung verschließen und untereinander möglichst stark fortpflanzen sollten. Von diesen Zweckgemeinschaften, „Biologische Völkerkeime“ genannt, versprach sich Thomsen auch ein „Hochzüchten wertvoller Erbanlagen“106. Über dem Ziel einer möglichst hohen „Quantität der Nachkommenschaft", so mahnte Thomsen, dürfe jedoch "nicht die Qualität derselben vergessen werden "107 - eine traditionsreiche, seit dem ausgehenden 19. Jahrhundert geradezu gebetsmühlenartig wiederholte Forderung der sogenannten „Positiven Eugenik" ${ }^{\prime 108}$, die von Thomsen jedoch in den spezifischen Argumentationskontext des angeblichen chinesischen Populationsdrucks gestellt wurde.

Im inneren Aufbau der „Völkerkeime“ unterschied Thomsen zwischen „Innengemeinde“, „Außengemeinde“ und "Schutzherren“. Die „Innengemeinde“ sollte sich „aus den zur Bildung des Völkerkeims zusammengetretenen ,Stammeltern“"

103 Ebd., S. 35.

104 Dieser fatalistische Zug im Denken Thomsens ist evident, vgl. ebd. S. 40: „Bei dem beginnenden modernen Völkersterben werden nach meinem Urteil die Chinesen wiederum ihr ganzes Volk retten und sogar noch an Zahl zunehmen. Alle übrigen werden aber, trotz aller Bemühungen - die man natürlich nicht aufgeben darf - trotz aller Eheverbote, aller Strafen für Empfängnisverhütung und Abtreibung, trotz aller Prämien auf Kinderreichtum usw. durch Mischehen und Geburtenrückgang zunächst zu je einem kleinen Rest zusammenschmelzen. Erst wenn es soweit gekommen ist, wird der kleine Rest sich - vielleicht - aufraffen und wieder anfangen zu wachsen oder wenigstens stationär zu bleiben“ (Herv. i. Orig.).

105 Thomsen, Völker, S. 45.

106 Ebd., S. 61.

107 Ebd., S. 43 (Herv. i. Orig.).

108 Pars pro toto sei auf den Arzt Wilhelm Schallmayer (1857-1919) verwiesen, der in seiner einflussreichen Preisschrift Vererbung und Auslese im Lebenslauf der Völker. Eine staatswissenschaftliche Studie auf Grund der neueren Biologie (1903) eine „stärkere Fortpflanzung der generativ wertvolleren Volkselemente“ verlangte bei gleichzeitig „möglichst geringe[r] Vermehrung der geistig und körperlich Schwächlichen“ (Schallmayer, Vererbung, S. 332, 340). 
konstituieren, die „Außengemeinde“ hingegen aus deren Kindern sowie aus „Unverheirateten “ und „Witwen und Witwern“, die „aus der Innengemeinde“ hervorgingen. Heirateten zwei Mitglieder der Außengemeinde, sollte ihnen Zutritt zur Innengemeinde gewährt werden. Eine „Fremdheirat" bedeute hingegen den Ausschluss aus dem „Völkerkeim“. Die „Schutzherren“ rekurrierten sich in der Vorstellung Thomsens schließlich aus Personen, die zwar in keine „der beiden Gemeinden [...] eintreten "109 könnten, den Völkerkeim aber gleichwohl fördern wollten.

Um den inneren Zusammenhalt der „Völkerkeime“ zu gewährleisten, schwebte Thomsen ein identitäts- und gemeinschaftsstiftender „Nachkommenkult" vor. Mitglieder der „Innengemeinde“ sollten „auf mindestens 4 Kinder in jeder Ehe“ verpflichtet werden, andernfalls sterbe „die Familie, bzw. das Volk“ notgedrungen aus. Die Tatsache, dass das strikte Fortpflanzungsmodell der „Innengemeinde" inzestuösen Beziehungen Vorschub leisten musste, entging Thomsen dabei nicht; doch versuchte er, diese Gefahr durch vage historische Analogien zu relativieren. ${ }^{110}$ Wirtschaftlich sollten die "Völkerkeime" wiederum möglichst autark sein, um ihre Abschottung nach außen sicherzustellen. Am „lebensfähigste[n]“ sei jener „Völkerkeim“, der „alle seine Lebensbedürfnisse selbst“ schaffe und „nur für seine Luxusbedürfnisse von der Außenwelt abhängig wäre"111.

Als eines der vornehmsten Ziele der „Völkerkeime“ gab Thomsen schließlich die „systematische“ und „zielbewußt auf bestimmte Eigenschaften gerichtete Züchtung" von Nachkommen aus. Entscheidend dafür, welche Eigenschaften in Zukunft herangezüchtet würden, werde „im großen und ganzen die Weltwirtschaft" sein. "Sehr verschiedene Anlagen“ könnten sich als nützlich erweisen. Thomsen dachte etwa an die Befähigung zur Besiedelung von Gegenden, in denen „beliebige Menschen nicht leben können“, beispielsweise „Tropen- und Polargegenden, Gebirge, Hochplateaus, sumpfige Niederungen usw. "112 Welche Eigenschaften und Merkmale auch gezüchtet werden würden, Sinn und Bestimmung, so Thomsen, würden die Menschen in den „Völkerkeimen“ gewiss finden. Bildreich malte sich Thomsen das ,viel erhebendere Gefühl“ aus,

„einem solchen zielbewußten Völkerkeime anzugehören, als einem Adels-, Patrizier- oder Bauerngeschlechte (vorausgesetzt, daß dies nicht ebenfalls achtungswerte züchtnerische [sic!] Ziele verfolgt). Und wenn diese Keime zu Völkern heranwachsen, denke ich es mir viel erhebender,

109 Thomsen, Völker, S. 46.

110 Vgl. ebd., S. 51: „Schallmayer erzählt, daß drei hochbegabte [...] englische Familien schon seit 10 Generationen unter sich geheiratet haben und zu einem tüchtigen Völkchen herangewachsen seien“. Diese drei Familien seien ein solcher Völkerkeim, „denn wenn die übrigen Angelsachsen durch Vermischung verschwinden sollten, wären sie allein imstande, das englische Volk fortzupflanzen [...] Außerdem erzählt die Geschichte von Geschwisterheiraten, die bei alten ägyptischen, bei einem altpersischen Herrscherhause und bei den Inka durch Generationen hindurch Sitte gewesen seien, ohne uns gleichzeitig von einer Degeneration jener Familien zu berichten“.

111 Ebd., S. 47 (Herv. i. Orig.).

112 Ebd., S. 64-66 (Herv. i. Orig.). 
einem solchen, sich zielbewußt weiter und höher züchtenden Volke anzugehören, als einem ,wildgewachsenen', welches sein Dasein irgendeinem historischen Zufalle verdankt!"113

Vortragstätigkeit Thomsens und WirkUng auf Grimm - Um seinen Thesen Gehör zu verschaffen, nutzte Thomsen die reichliche Zeit, die ihm sein Ruhestand bot, auch immer wieder für Vorträge. Dass er dabei nicht ohne Erfolge blieb, dürfte sowohl seinem akademischen Rang als auch der allgemeinen Virulenz bevölkerungspolitischer Themen in der Weimarer Republik geschuldet sein. ${ }^{114}$ Allein für das knappe Zeitfenster zum Jahreswechsel 1928/29, in dem Thomsen mit Grimm korrespondierte, lassen sich vier Vorträge belegen: Zunächst sprach Thomsen am 4. Januar 1929 in seiner Heimatstadt Hannover auf einer Veranstaltung des Heimatbunds Niedersachsen ${ }^{115}$, anschließend referierte er zum Thema „Der Untergang des Abendlandes und die Bewegung der Kinderreichen“ im Auditorium Maximum der Universität Göttingen, dann erwähnte Thomsen im März 1929 eine „Ansprache an die Kinderreichen auf dem Göttinger ,Muttertage“ - gemeint war hier der Reichsbund der Kinderreichen Deutschlands. ${ }^{116}$ Darüber hinaus ist eine „Besprechung mit dem hannoverschen Adel um Celle“117 belegt, was angesichts dessen, dass sich Thomsen in seinen Schriften gerade auch an die gesellschaftlichen Eliten wandte, nicht überrascht.

Die Inhalte des Vortrags für den Heimatbund Niedersachsen lassen sich über den Nachlass Grimms detailliert rekonstruieren, da Thomsen dem Dichter im Vorfeld seiner Rede die Forderungen auseinandersetzte, die er in Hannover erhob und zur Diskussion stellte. Offenbar versprach sich Thomsen viel von dem kritischen Input Grimms. Thomsen eröffnete seinen Forderungskatalog mit dem Aufruf, alle niedersächsischen „,Heimat'- u[nd] ähnlichen Vereine“ zusammenzufassen und die öffentliche Wissensvermittlung über den „Mensch der Heimat“ (I) zu forcieren. Vor allem das angeblich drohende „Aussterben des ,Menschen der Heimat“ und seine „ev[entuellen] Rettungsmöglichkeiten“ sollten dabei behandelt werden. Was nämlich nütze „die ganze Heimatbewegung, wenn der alte Nieder-

113 Ebd., S. 75.

114 Vgl. hierzu: Weipert, „Mehrung der Volkskraft“; Ders., Wiederaufstieg; ferner die Beiträge in: Mackensen (Hg.), Bevölkerungslehre.

115 Vgl. DLA, A:Grimm, Andreas Thomsen an Hans Grimm, 3. Januar 1929. Für Informationen zum Heimatbund Niedersachsen, der 1901 in Hannover gegründet wurde und bis zum Ersten Weltkrieg auf etwas mehr als 1000 Mitglieder anwuchs, vgl. Hartung, Zivilisationskritik, S. 78-85.

116 Vgl. Weingart/Kroll/Bayertz, Rasse, S. 232-237. Der Bund umfasste bereits im September 1922, dem Jahr seiner Gründung, etwa 42000 Familien mit durchschnittlich 6,6 Kindern. Seit Juni 1923 trug er den kompletten, offiziellen Titel: Reichsbund der Kinderreichen Deutschlands zum Schutze der Familie. Er forderte „neben gesetzgeberischen Maßnahmen [...] eine familiengerechte Besteuerung, die Zuweisung menschenwürdiger Wohnungen sowie die Vergabe von Siedlungen und Pachtland an Kinderreiche“ (ebd., S. 233). Von dem Zuspruch der Bevölkerung her betrachtet war der Bund zwar ein Erfolg - 1930 besaß er rund 1200 Ortsgruppen -, ein großer Einfluss auf die Weimarer Familienpolitik blieb ihm jedoch verwehrt.

117 Vgl. DLA, A:Grimm, Andreas Thomsen an Hans Grimm, 30. März 1929. 
sachse, Friese, Allemanne [sic!] usw. daraus wegstirbt u[nd] durch die furchtbaren Polen usw. ersetzt wird?!" Weiterhin forderte Thomsen die sofortige Gründung ,eines ,städtischen“ und „eines ,dörflichen V[ölkerkeim]s“" in Hannover (II/III) sowie in der „Tochterstadt ,Hannover in den Ver[einigten] Staaten “" (IV/V). Hierzu sollte ein „Dutzend biologisch einwandfreier tüchtiger kinderreicher Kalenberger Bauernfamilien" nach Amerika entsendet werden (VI), indes „auf dortige Kosten“. Darüber hinaus bekräftigte Thomsen einen „vom Bund der Kinderreichen, vom Deutschen Schutzbund usw." ausgehenden Vorschlag, die Tiroler Bevölkerung gesammelt ,in einer Gebirgsgegend anzusiedeln“ (VII). Des Weiteren sollten dörfliche „Völkerkeime“ in der Lüneburger Heide (VIII) und an jenen Orten, „wo noch altfränkisch gesprochen" werde (IX), gebildet werden. Dieselbe Forderung galt für das „,1000jährige Bauerngeschlecht' bei Lehrte“ (X). Zuletzt forderte Thomsen den „Zusammenschluß alter Hannoverscher Familien zur gegenseitigen Versicherung auf Kinderreichtum"118 (XI).

Grimm reagierte auf Thomsens Ausführungen positiv. Zufolge einer Notiz in dem nächsten Brief Thomsens ging Grimm einzig mit dem Vorschlag einer kollektiven Umsiedelung der Tiroler Bevölkerung „nicht [...] eins“. Thomsen bot dem Dichter daher an, „die Sache" bei nächster Gelegenheit nochmals zu „besprechen"119. Vor allem mit dem Szenario einer bevorstehenden „Slawisierung" der europäischen „Kulturvölker“ und der Gefahr ihrer rassischen „Entedelung" rannte Thomsen bei Grimm offene Türen ein, beschwor dieser doch zeit seines Lebens die Gefahr eines kulturell und rassenbiologisch nivellierenden Eindringens „östlicher" Völker in Deutschland und Europa. Noch nach dem Zweiten Weltkrieg behauptete Grimm in seinen energischen Versuchen, zur Ehrenrettung des „Dritten Reichs“ beizutragen, dass die Nationalsozialisten durch das Unternehmen Barbarossa einer unmittelbar bevorstehenden Invasion der Sowjetvölker zuvorgekommen seien. ${ }^{120}$

Dass Grimm von Thomsens Szenario eines bis zum Jahr 2200 alles dominierenden chinesischen Volks sonderlich beeindruckt wurde, ist hingegen unwahrscheinlich. Thomsens strikt hierarchisierende Differenzierung zwischen a priori hochbegabten und unbegabten Völkern ${ }^{121}$ entsprach jedoch wieder ganz den politisch-ideologischen Gewissheiten des Dichters. Grimms Denken ging „immer

118 DLA, A:Grimm, Andreas Thomsen an Hans Grimm, 3. Januar 1929 (Herv. i. Orig.).

119 DLA, A:Grimm, Andreas Thomsen an Hans Grimm, 30. März 1929.

120 Vgl. Kap. 6.2.

121 Ihre überlegenen Anlagen, so glaubte Thomsen, werde den höher begabten „Kulturvölkern“ aufgrund ihrer niedrigen Reproduktionsrate langfristig nichts nutzen. Durch ein einfaches Gedankenexperiment versuchte Thomsen dies zu illustrieren: „Nehmen wir einmal an, 10000 Engländer und 10000 Neger wären verurteilt, in alle Ewigkeit miteinander auf einer Insel fortzuleben. Mischen sie sich und entsteht ein Mulattenvolk, welches begabter als das der Neger, aber unbegabter als das der Engländer ist, so verstößt das Gesamtwohl der Inselbevölkerung. Denn wenn die Engländer auch nur zu einem kleinen Bruchteil sich dort rein erhalten, so ist der ganze Kulturzustand der Insel ein höherer. Auch für die Neger ist dieser Zustand vorzuziehen, vor allem in Zeiten der Not oder der Gefahr, deren ein Engländerhirn eher Herr werden kann als ein Negerhirn" (Thomsen, Völker, S. 33). 
von einer naturgegebenen Ungleichwertigkeit der einzelnen Kulturen und Völker“ aus, er lehnte daher alle „egalitären Modelle der Gleichheit der Menschen und Nationen $\mathrm{ab}^{\text {"122 }}$. Darüber hinaus finden sich bei Grimm durchaus Anleihen an die Denkfiguren der Eugenik ${ }^{123}$, wie sie in Thomsens Text zum Ausdruck kommen. Im April 1939 betonte Grimm in einem anlässlich von Hitlers 50. Geburtstag verfassten Text, dass es bei der Umsetzung des „Willen[s] zu einem ganz neuen Menschentume“ nicht mehr ausreiche, sich auf „die alten Lehren und Erkenntnisse“ zu stützen. Zwar seien diese „nicht falsch“, doch würden sie der Gegenwart nicht mehr Genüge tun, zumal die Welt unter ihnen „unversehens vor eine wahnwitzige Not geraten“ sei. Gemeint war damit die vermeintliche gesellschaftliche „Vermassung“, die schon in Volk ohne Raum ein zentrales Motiv war: „Menschenhorden“, so Grimm, „entstanden und entstehen und machen die ratlose Erde häßlich und fressen sie kahl“. Aus einem „falsch begriffenen Evangelium“ heraus, so Grimm, habe eine mittlerweile überkommene Weltsicht irrigerweise „jedes Einzelleben, ob gesund oder krank, als geheiligt“ angesehen. Im Gegensatz dazu setze der Wille zum „neuen Menschen“ auf die

„verpflichtete Auswahl an Stelle der Horde und auf das Vorrecht der Auswahl, die gesund und geradegewachsen ist und gesund und geradegewachsen denkt und gesund und geradegewachsen fühlt, und die also nicht lügt aus Bequemlichkeit [...], sondern die zum großen Gotte ehrfürchtig aber Auge in Auge zu stehen vermag, wenn sie das neue Gesetz von ihm endlich empfängt“124.

\section{3 „Rassegedanke" und „Völkischer Gedanke" bei Adalbert Wahl}

Biografie und Wissenschaftliches Profil Adalbert WaHLS Und SEIN VerhäLtNis ZU KolBeNHEYER - Adalbert Wahl, am 29. November 1871 in Mannheim geboren, studierte Geschichtswissenschaft an den Universitäten Oxford, Berlin, Wien und Bonn. Hatte er seine Promotion an der Universität Bonn noch einem frühneuzeitlichen Thema gewidmet ${ }^{125}$, wandte er sich seit seiner Habilitationsschrift Studien zur Vorgeschichte der Französischen Revolution (1901) ganz der Neueren Geschichte zu. Seit 1905 Extraordinarius in Freiburg im Breisgau wurde Wahl 1908 zum ordentlichen Professor für Neuere Geschichte am Hamburgischen Kolonialinstitut berufen. Zwei Jahre später erhielt Wahl - nachdem Friedrich Meinecke zuvor einen Ruf abgelehnt hatte ${ }^{126}$ - den Lehrstuhl für Neuere Geschichte an der Universität Tübingen. ${ }^{127}$ Mit einer einjährigen Unterbrechung an der estländischen Universität Dorpat im Zeitraum 1918/19 lehrte Wahl bis zu

122 Gümbel, Volk, S. 71.

123 Vgl. die Hinweise zu Grimms Handbuch Afrikafahrt West (1913) in Kap. 2.1.

124 Dem Führer. Worte deutscher Dichter zum Geburtstag des Führers 1941, S. 54 f.

125 Adalbert Wahl, Compositions- und Successions-Verhandlungen unter Kaiser Matthias während der Jahre 1613-1615, Bonn 1895.

126 Vgl. Meinecke, Werke, Bd. 10, S. 167, Anm. 3.

127 Vgl. Daniels, Geschichtswissenschaft, S. 280. 
seiner Emeritierung im Jahr 1938 in Tübingen. Zwischen 1921 und 1922 amtierte er als Rektor der Universität.

Bis zu seiner Berufung nach Tübingen hatte Wahl sein geschichtswissenschaftliches Werk ganz der Französischen Revolution gewidmet. Ernst Schulin hat Wahl in diesem Zusammenhang als „den eigentlichen und typischen deutschen Revolutionshistoriker vom Wilhelminischen Kaiserreich bis zur Hitlerzeit" porträtiert, der in seinen Kollegs „über den Prozeß und die Hinrichtung Ludwigs XVI. und Marie Antoinettes nur unter Tränen" 128 habe erzählen können. Wie wörtlich diese Anekdote zu nehmen ist, kann dahingestellt bleiben. Ohne Zweifel war Wahl aber ein führender Vertreter jener deutschen Historikerschule, der die Französische Revolution schlechterdings als die Urkatastrophe des (langen) 19. Jahrhunderts galt. ${ }^{129}$

Bereits im späten Kaiserreich war Wahl einer der „bekanntesten Vertreter“ einer „ultranationalistischen, expansionistischen, antidemokratischen Richtung“130 in der deutschen Historikerzunft. Es verwundert daher nicht, dass der Tübinger Historiker mit der NS-Bewegung bereits vor dem Aufstieg der NSDAP zur Massenpartei in Berührung kam; eine 1929 veröffentlichte Meldung der Nationalsozialistischen Monatshefte belegt Wahls Mitgliedschaft im Förderkreis von Alfred Rosenbergs KfdK. ${ }^{131}$ Zwei Jahre später zählte Wahl - gemeinsam mit Kolbenheyer und dem 1929 an die Universität Tübingen berufenen, völkisch orientierten Philosophieprofessor Max Wundt ${ }^{132}$ - zu den Gründungsmitgliedern der Tübinger Ortsgruppe des KfdK.133 Eine maßgebliche Schnittmenge mit der NS-Ideologie bildete Wahls rabiater, über den nationalkonservativen Basiskonsens hinausgehender Antiparlamentarismus. In seinen wissenschaftlichen Arbeiten skizzierte Wahl das parlamentarische System als einen Hort politischer „Entartung und

128 Schulin, Revolution, S. 12.

129 Paradigmatisch kommt dies im Vorwort des ersten Bandes seiner Deutschen Geschichte seit 1871 zum Ausdruck, vgl. Wahl, Deutsche Geschichte, Bd. 1, S. VII. Dort skizziert Wahl die Wirkungen der Französischen Revolution wie folgt: „Es ist unverkennbar, daß in zahlreichen europäischen Staaten seit und infolge der französischen Revolution ein Abstieg einsetzt, von dem freilich noch nicht klar ist, wie tief hinab er führen wird“. Dem seit $1789 \mathrm{zu}$ beobachtenden Anstieg der materiellen Reichtümer stehe ein „weit größerer Verlust gegenüber“. Überall wo die Französische Revolution „siegreich“ vorgedrungen sei, habe sie „die letzten Grundlagen der Kultur und der Leistungen der Völker angefressen: so die Einheitlichkeit des Volksempfindens [...] und sie hat überall das Gefühlsmäßige im menschlichen Leben zu Gunsten von rechnenden Nützlichkeitserwägungen zurückgedrängt und vor allem - das Wichtigste - dem Glauben zu Gunsten des reinen Verstandes einen unerbittlichen Krieg erklärt. Durch all dies hat sie vor allem Kunst und Literatur einen schweren, vielleicht tödlichen Schlagversetzt [sic!]“.

130 Iggers, Geschichtswissenschaft, S. 320.

131 Vgl. Bollmus, Amt, S. 28.

132 Knappe Hinweise zu Person und Wirken von Max Wundt in: Dahms, Philosophen, S. 729738.

$133 \mathrm{Zu}$ Kolbenheyers Beziehung zum KfdK vgl. Kap. 5.1; Christian Tilitzki vermutet, dass die Berufung Wundts nach Tübingen im Oktober 1929 maßgeblich der „Regie“ Wahls zuzuschreiben ist, vgl. Tilitzki, Universitätsphilosophie, S. $283 \mathrm{f}$. 
Korruption“134. In seinen Vorlesungen machte Wahl nach 1918 derart rigoros gegen die politische Ordnung der Republik Stimmung, dass sich der württembergische Landtag im März 1921 kritisch mit der Wirkung des polemisierenden Historikers auseinandersetzte. ${ }^{135}$ Die entsprechende, berechtigte Debatte blieb jedoch ohne nachhaltige Wirkung. Seine Haltung zur Republik unterstrich Wahl nicht zuletzt auch dadurch, dass er öffentlich die Abgeordneten des Reichstags beleidigte; „seit der Bismarckzeit“ hätten diese an „inneren Werte immer mehr"136 abgenommen. Diese Behauptung war indes nicht originell, sie findet sich in vielen rechtsradikalen Traktaten der Zeit, etwa in Heinrich Claß' Wenn ich der Kaiser wär'. 137

Da sich Wahl und Kolbenheyer während der Tübinger Jahre des Dichters (1919-1932) bei Bedarf jederzeit persönlich sprechen konnten, lässt sich ihre Beziehung durch die Nachlassüberlieferung nur bruchstückhaft erfassen. Die hohe gegenseitige Wertschätzung steht indes außer Zweifel: Als Stapel im Frühjahr 1929 zu einem Vortrag nach Tübingen reiste, gehörte Wahl zu jenen Professoren, mit denen Kolbenheyer Stapel unbedingt bekannt machen wollte. ${ }^{138}$ Die persönliche Verbundenheit des Historikers gegenüber dem Dichter kommt in einem Brief Wahls an Kolbenheyer vom 11. Dezember 1931 zum Ausdruck, in dem Wahl die Bekanntschaft mit Kolbenheyer „zu den schönsten Errungenschaften“ seines „letzten Lebensjahrzehnts“139 rechnete. Wahl hatte die Werke des Dichters demnach „schon seit Jahren mit höchster Bewunderung und Dankbarkeit“ verfolgt. Tübinger Gespräche mit Kolbenheyer - in denen er „mächtig aufgerüttelt“ worden sei „durch das was Sie sagten und wie Sie es sagten“ - waren dem Historiker „unvergesslich“140. Mit Blick auf den damals bevorstehenden Umzug Kolbenheyers nach Solln bei München ${ }^{141}$ hoffte Wahl schließlich, dass an den „Gerüchten nichts dran“ wäre, Kolbenheyer werde „Tübingen demnächst verlassen“. „Sie sind uns unersetzlich" 142 . Nach dem erfolgten Umzug der Familie Kolbenheyer blieb es schließlich bei einigen wenigen Briefen Wahls - meist Danksagungen für die Zusendung von Freiexemplaren neuer Veröffentlichungen des Dichters. Der Kontakt bracht 1942 endgültig ab. Bis zu dem Tod des Historikers am 5. März 1957 in Tübingen sind keine weiteren Briefe überliefert.

134 Zitiert nach: Langewiesche, Politikstile, S. 3.

135 Vgl. Paletschek, Erfindung, S. 503, Anm. 135.

136 Zitiert nach: Biefang, Seite, S. 276.

137 Vgl. Frymann, Kaiser, S. 44: „das Niveau der Volksvertretung sinkt, und damit werden die Abgeordneten auf der bürgerlichen Seite untauglicher, den Kampf gegen eine unfähige Regierung und gegen die Sozialdemokratie zu führen. Heute ist kein Zweifel mehr möglich, daß der Reichstag keine Volksvertretung ist, der die Achtung der staatserhaltenden Kreise des Volkes zufällt. Die Auslese auf der bürgerlichen Seite hat so gelitten, daß die politischen Köpfe zu zählen sind, womit das parlamentarische Tohuwabohu erklärt ist.“

138 Vgl. DLA, A:Stapel, Erwin Guido Kolbenheyer an Wilhelm Stapel, 22. Januar 1929.

139 KAG, Adalbert Wahl an Erwin Guido Kolbenheyer, 11. Dezember 1931.

140 Ebd. (Herv. i. Orig.).

141 Vgl. Kap. 3.4.

142 KAG, Adalbert Wahl an Erwin Guido Kolbenheyer, 11. Dezember 1931. 
Der „RAssegedanke“ in Wahls Hauptwerk „Deutsche Geschichte“ - Die sich auf rund 2800 Seiten erstreckende, vierbändige Gesamtdarstellung Deutsche Geschichte von der Reichsgründung bis zum Ausbruch des Weltkriegs (erschienen zwischen 1926 und 1936 im Stuttgarter Kohlhammer-Verlag) darf als das Hauptwerk Adalbert Wahls gelten. ${ }^{143}$ Wahls Gesamtdarstellung fand sowohl im akademischen Milieu als auch in der rechten Publizistik wohlwollende Aufnahme. Nach der Publikation des dritten Bands im Jahr 1932 wurde das Werk in der Monatsschrift Deutschlands Erneuerung, einem Hauptorgan der völkischen Bewegung, von dem antisemitischen Musik- und Kulturhistoriker Hermann Seeliger ${ }^{144}$ als die „schlechthin beste und lichtvollste Darstellung dieses Zeitraums“ und „eines unserer besten Geschichtswerke überhaupt" bezeichnet. ${ }^{145}$

Ein Blick in das inhaltlich weit über bloße Politikgeschichte hinausgehende Werk offenbart rasch das hohe Gewicht, das der Tübinger Historiker dem „Rassegedanken“ zumaß. Wahl gründete einerseits Teile seiner eigenen Argumentation auf rassistisches Gedankengut, andererseits schildert er den „Rassegedanke“ auch als Teil des wissenschaftshistorischen Rahmens seines Untersuchungszeitraums. Wahl war dabei jedoch merklich darum bemüht, sich von undifferenzierten und fanatischen Rasse-Phantasten $\mathrm{zu}$ distanzieren, indem er ihre „unwissenschaftliche“ Herangehensweise kritisierte. Durch diese hätten sie den „Rassegedanken“, den Wahl ausdrücklich als „höchst bedeutend“ gelten ließ, trivialisiert. Ähnlich wie Felix Krueger wollte auch Wahl keine rein auf „körperliche Rassenmerkmale“ beschränkte, monokausale Perspektive gelten lassen, sondern betonte die gleichzeitige Bedeutung „geistig-seelische[r]“ Rasse-Spezifika. ${ }^{146}$ Das konkrete Verhältnis zwischen der biologischen und seelischen Dimension des Rasse-Begriffs blieb dabei jedoch völlig im Dunkeln.

Um sich von den wissenschaftsfernen und -feindlichen Segmenten der völkischen Bewegung abzugrenzen, verwies Wahl auf das Negativbeispiel des schwärmerischen Germaneneuphorikers Ludwig Woltmann. ${ }^{147}$ Dem von „irrationale[m], methodisch unkontrollierte[m] Fabulieren “148 ge(kenn)zeichneten historischen Werken Woltmanns erteilte Wahl eine Absage. So lehnte er etwa die Auffassung des Laienhistorikers, wonach nichtgermanische Völker niemals "große Männer“

${ }^{143}$ Eine ausführliche, aufgrund des Verzichts auf historische Kontextualisierung leider stark deskriptive Zusammenfassung der vier Bände bietet: Daniels, Geschichtswissenschaft, S. 280300.

144 Eines der persönlichen Hauptanliegen Seeliger war es, Richard Wagner als einen völkischen Vorkämpfer und Wegbereiter des „Dritten Reichs“ darzustellen, vgl. die zahlreichen Hinweise in: Hein, „Es ist viel ,Hitler' in Wagner“.

145 Seeliger, Geschichtsschreibung, S. 96. Seeliger ordnete die von ihm untersuchten Studien nach den Kategorien „unerschrockenes Deutschbewußtsein“ einerseits und „weltbürgerlichutopistische Denkweise von vielfach mimosenhafter Zagheit" andererseits. Adalbert Wahl galt Seeliger in diesem Zusammenhang als mustergültiger Vertreter der ersten Kategorie.

146 Wahl, Geschichte, Bd.3, S. 53; zu Felix Kruegers Einstellung zum „Rassegedanken“ vgl. Kap 4.1.

$147 \mathrm{Zu}$ Werk und Person Woltmanns (1871-1907) vgl. die zahlreichen Hinweise in: Schütz, Rassenideologien.

148 Sieferle, Rassismus, S. 443. 
hervorbrächten, als zu undifferenziert ab. Zugleich lobte er es jedoch als „verdienstlich“, dass Woltmann in seinen Werken nachgewiesen habe, dass „,besonders in den höheren Schichten Italiens und Frankreichs [...] viel germanisches Blut“ fließe - wie der „körperliche Befund“ erwiesen habe. ${ }^{149}$ Insgesamt zog er ein versöhnliches Fazit: Trotz zahlreicher „Übertreibungen und Kritiklosigkeiten“ habe sich Woltmann das bleibende Verdienst erworben, mit „dazu beigetragen“ zu haben, dem „Rassegedanke[n]“ höhere öffentliche Aufmerksamkeit angedeihen zu lassen. ${ }^{150}$ Im Kern blieb Wahls kritische Distanz zur völkischen Rasseideologie also auf die Ablehnung ihrer ungebildetsten und dilettantischsten Exponenten beschränkt, insbesondere hinsichtlich ihrer Gleichgültigkeit gegenüber (geschichts-)wissenschaftlichen Standards. Dies zeigt auch der Sachverhalt, dass etwa Houston Stewart Chamberlain, einer der „wichtigsten Wegbereiter der völkischen Weltanschauung"151, in Wahls Schriften trotz Detailkritik mit offener Sympathie behandelt wird. Entsprechend viel Raum räumte er dem Bayreuther Rasseideologen in seiner Darstellung ein. ${ }^{152}$

Schon in dem 1926 erschienenen ersten Band seiner Deutschen Geschichte stützte sich Wahl mehrfach auf „Rasse“ als analytische Kategorie. Für Kenner des Autors kam dies nicht überraschend: Bereits 1913 hatte Wahl in einer Festrede zum 100-jährigen Jubiläum der Völkerschlacht bei Leipzig ${ }^{153}$ verkündet, dass „über der Nation [...] als natürlich gegebene Grundlage der Organisation die Rasse“154 stehe. Dreizehn Jahre später skizzierte Wahl als Einstieg in seine Deutsche Geschichte den angeblichen „rassischen“ Zustand des deutschen Volks im Jahr 1871. Die Leser der Deutschen Geschichte erfuhren dabei, dass eine historische Betrachtung des Kaiserreichs von dem Sachverhalt auszugehen habe, dass nach der Gründung des Kaiserreichs im deutschen Volk „noch immer das nordische Blut“ überwogen habe, im Norden des Reichs „freilich [...] weit mehr als im Süden“. Laut Wahl konnte auch für den unvoreingenommenen Betrachter, der sich von „Uebertreibungen“ freihalte, kein Zweifel bestehen, dass die „nordische Rasse das Salz der Erde“ sei - „körperlich und geistig hochbegabt“, über alle „andere[n] Rassen weit hinaus"155. Schon im Einstieg der Deutschen Geschichte lässt sich also die Akzeptanz und Verbreitung völkischer Ideologeme unter dem Vorzeichen eines vor „Übertreibungen“ warnenden, bildungsbürgerlichen Mäßigungsgestus nachweisen, wie er auch für Grimm, Kolbenheyer und Stapel charakteristisch war. ${ }^{156}$

149 Wahl, Geschichte, Bd. 3, S. 53f. Konkret erwähnt Wahl hier folgende Werke Woltmanns: Die Germanen und die Renaissance in Italien (1905) und Die Germanen in Frankreich. Eine Untersuchung über den Einfluss der germanischen Rasse auf die Geschichte und Kultur Frankreichs (1907).

150 Wahl, Geschichte, Bd. 3, S. 54.

151 Puschner, Bewegung, S. 280.

152 Vgl. Wahl, Geschichte, Bd. 3, S. 54-63.

153 Vgl. hierzu einführend Poser, Jahrhundertfeier; Dyroff, Jahrhundertfeiern.

154 Zitiert nach: Weber, Tendenzen, S. 852.

155 Wahl, Geschichte, Bd. 1, S. 37.

156 Vgl. Kap. 2.2.2. 
Im deutschen Süden, so fuhr Wahl fort, sei es im Lauf der Geschichte zu „starke[n] Blutbeimischungen aus anderen Rassen“ "gekommen, insbesondere aus der „ostischen“ Rasse, „wie man heute sagt" ${ }^{157}$. Diese Bemerkung war eine Referenz auf die seinerzeit populäre, erstmals 1922 erschienene Rassenkunde des Deutschen Volkes von Hans F. K. Günther, der 1930 - ohne annähernd ausreichende wissenschaftliche Qualifikation - auf Veranlassung des nationalsozialistischen thüringischen Innen- und Volksbildungsministers Wilhelm Frick einen neu eingerichteten Lehrstuhl für „Sozialanthropologie“ an der Universität Jena erhalten sollte. ${ }^{158}$ Günther unterschied in seinen Arbeiten insgesamt sechs europäische „Systemrassen“, namentlich die „nordische“, „westische“, „ostische“, „dinarische“, „fälische“ und schließlich die „ostbaltische“ Rasse. ${ }^{159}$ Ganz im Sinne Günthers deutete Wahl die „ostische“ Rasse als biologisch a priori minderwertig. Folglich habe die Rassenvermischung im deutschen Süden ein insgesamt weniger begabtes „Menschenmaterial“ hervorgebracht, das dafür „unendlich viel leichter zu regieren" gewesen sei. Um es sich mit seinem bajuwarischen Publikum nicht zu verscherzen, fügte Wahl indes versöhnlich hinzu: „Tüchtig aber waren auch die Deutschen dieses Blutes“. Der Grad der Tüchtigkeit galt ihm dabei freilich als direkt proportional zur „Beimischung“ nordischen „Blutes“. Darüber, dass das deutsche Volk „als Ganzes“ betrachtet „im Vergleich mit andere[n] Völker[n]“ die mit Abstand höchste „Begabung " 160 besaß, ließ der Tübinger Neuzeithistoriker keine Zweifel gelten.

Auch in Wahls vergleichender Betrachtung der 1871 „neu erworbenen und völkisch gemischten Gebiete" im deutschen Osten und Westen spielte rassistisches Denken eine zentrale Rolle. Dem ost-westlichen Vergleich widmete Wahl ein ausführliches Kapitel, in dem er diametrale Gegensätze zwischen beiden Grenzregionen bilanzierte. ${ }^{161}$ Als Vergleichsgrundlage dienten ihm dabei Elsass-Lothringen und Posen, wo Polen vor 1918 die deutliche Bevölkerungsmehrheit stellten. ${ }^{162}$ Der „Bevölkerung mit uralter deutscher Hochkultur“ im Westen stellte Wahl ein kulturelles Brachland im Osten gegenüber, das ,in geistiger, materieller, sittlicher, wirtschaftlicher Hinsicht" seit jeher tief unter Deutschland gestanden habe. Der Deutsche im Osten verliere nie den Eindruck, „auf einem Kulturboden schwach

157 Wahl, Geschichte, Bd. 1, S. 37.

158 Während zu Leben und Werk Hans F. K. Günthers bis dato vergleichsweise wenige Forschungen vorgelegt worden sind, darf die Berufung Günthers zum ordentlichen Professor die an der dortigen Universität wie im gesamten akademischem Deutschland starke Proteste hervorrief - als gut erforscht gelten. Vgl. Hoßfeld, Jahre; Kaudelka, Berufung.

159 Zusammenfassende Hinweise zu Günthers Rassekonzeptionen in: Freisleben, Grundelemente, S. 51-81.

160 Wahl, Geschichte, Bd. 1, S. 37f. (Herv. i. Orig.).

161 Ebd., S. 286-341.

162 Zwischen 1871 und 1910 stieg der polnische Anteil an der Gesamtbevölkerung Posens von $61 \%$ auf $64,7 \%$ an, während der deutsche Bevölkerungsanteil leicht von 35,1\% auf 34\% sank. Die Gesamtbevölkerung Posens betrug 1871 etwa 1584000 , 1910 etwa 2100000 Menschen. Vgl. Ferrari Zumbini, Wurzeln, S. 471. 
wie dünnes Eis zu stehen" 163 . Vor diesem Hintergrund postulierte Wahl, dass das „elsaß-lothringische Problem“ - gemeint war hier die Frage einer funktionalen Eingliederung des „Reichslands“ Elsass-Lothringen in das Deutsche Reich - nach 1871 lösbar gewesen sei, habe es sich bei der dortigen Bevölkerung doch „um ein im Wesentliches deutsches Volk“ gehandelt. Demgegenüber seien die „volks- und rassefremd[en]“ Polen durch keine Anstrengungen zu integrieren oder „bekehren“ gewesen. Allenfalls durch eine „sehr starke Einwanderung von altdeutschen Elementen“, so Wahl, „wäre die polnische Frage lösbar gewesen“164. Er griff damit die schon im wilhelminischen Kaiserreich populäre Idee der „Inneren Kolonisation"165 auf, die in den Augen vieler völkischer Ideologen nach 1871 in nur völlig unzureichendem Maße politisch verfolgt worden sei. Bevölkerungs- und rassebedingt habe Posen im Kaiserreich so ein Fremdkörper bleiben müssen.

Jüdische Typologie in Wahls „Deutscher Geschichte“ - Um eine detaillierte Erläuterung der Stellung der Juden in der Kaiserreichsgesellschaft bemühte sich Wahl in seiner Gesamtdarstellung nicht. Judentum und „Judenfrage“ spielen in der Deutschen Geschichte nur eine Nebenrolle. Die wenigen Passagen, in denen sich Wahl zu diesen Themenkomplexen äußert, lehnen sich indes deutlich an antisemitische Topoi an. Dies beweist unter anderem die Beschreibung einzelner jüdischer „Typen“ in Wahls Text. Ein sprechendes Beispiel ist die Charakterisierung Eduard Laskers, der 1867 die Nationalliberale Partei mitbegründet hatte und ab 1880 bis zu seinem frühen Tod 1884 zur maßgeblichen Figur der Liberalen Vereinigung avanciert war. ${ }^{166}$ Lasker galt Wahl schon aufgrund seiner kritischen Haltung zu Bismarck als „der böse Geist der Nationalliberalen“. Den Lesern wird er als ein „winziges Männlein [...] mit stark jüdischer Physiognomie“ vorgestellt, das sich jeglichem Lebensgenuss verschlossen habe und gänzlich in der Arbeit aufgegangen sei. ${ }^{167}$ Schon die frühen Schriften Laskers, so Wahl, würden zwar eine „spezifisch dialektische Begabung“ offenbaren, ansonsten jedoch - „wie so viele Werke semitischer Autoren“ - eine „blendende Klarheit“ lediglich „vortäuschen“. Laskers „Fähigkeit, mit vielen Worten wenig zu sagen“, deutete der Tübinger Historiker als ein spezifisches Merkmal jüdischer Autoren, die immer dann durch „verschwommen[e] und lebensfern[e]“ Ӓußerungen in Erscheinung träten, sobald es nicht mehr nur um Fragen des „Ausdruck[s]“, sondern um die „Dinge, die zu Grunde“ liegen, gehe. ${ }^{168}$ Oberflächlichkeit und ein substanzloser, lebensferner Intellektualismus waren es also, die Lasker in der Darstellung Wahls zu einem typisch jüdischen Politiker und Publizist machten.

163 Wahl, Geschichte, Bd. 1, S. 323.

164 Ebd., S. 324, 327.

165 Vgl. Helmes, Kolonisation; Weipert, Siedlung.

$166 \mathrm{Zu}$ Eduard Lasker vgl. v. a. Harris, Study; Dill, Parlamentarier. Zur Liberalen Vereinigung, die sich vor der Reichstagswahl am 28. Oktober 1884 mit der Deutschen Fortschrittspartei zur Deutsch-Freisinnigen Partei zusammenschloss, vgl. Cioli, Pragmatismus, S. 148-189.

167 Wahl, Geschichte, Bd. 1, S. 49.

168 Ebd., S. 49f. 
Ein weiteres Beispiel antisemitisch konnotierter Personenbeschreibung ist Wahls Porträt des sogenannten „Eisenbahnkönigs“ Bethel Henry Strousberg, der während der „Gründerzeit“ zu einem überaus erfolgreichen Großunternehmer aufstieg, Mitte der 1870er Jahre jedoch aufgrund krimineller Machenschaften inhaftiert wurde und wenig später Konkurs machte. ${ }^{169}$ Für ebenjene Machenschaften war Strousberg von niemand anderem als Eduard Lasker am 7. Februar 1873 in einer dreistündigen Reichstagsrede angegriffen worden, wobei Lasker zugleich offenlegte, dass „hochrangige Politiker und Beamte, darunter Hermann Wagener, langjähriger Berater Bismarcks, bei der Vergabe von Eisenbahnkonzessionen, von denen sie profitierten, ungesetzliche Praktiken zugelassen hatten"170. In der ansonsten so detailverliebten Darstellung Wahls wird diese mutige und verdienstvolle Rede Laskers bezeichnenderweise nicht erwähnt. Stattdessen erscheint der „ostpreußische Jude“ Strousberg als ein autonom agierender „Hauptverderber der guten geschäftlichen Sitte"171 des deutschen Volks. Strousberg - der von sich selbst behauptet habe, „aus vornehmer alter Judenfamilie“ zu stammen und nicht etwa „Abkömmling schmieriger Ostjuden“ zu sein - besaß nach der Auffassung Wahls in geschäftlichen Dingen zwar „ein kluges Auge mit ruhigem Blick.“ Dieser habe jedoch keinem „Menschenkenner [...] Vertrauen“ eingeflößt. Die „wichtigsten Anregungen“ für seine „geschäftlichen Methoden“ habe Strousberg in England erworben - ein laut Wahl symptomatischer Sachverhalt, pflege sich die jüdische „Rasse [doch] nicht durch Originalität auszuzeichnen“172. Insgesamt gestand Strousberg zwar zu, sich „um das Eisenbahnnetz Deutschlands hoch verdient gemacht“ zu haben, ohne sich jedoch die vielsagende Andeutung sparen zu können, dass Strousberg als Unternehmer im Kern von „Ruhm- und Herrschsucht“ angetrieben worden sei sowie von „jene[m] semitische[n] Tätigkeitsdrang, den wir alle kennen" 173 .

"Jüdische" Wesenszüge schrieb Adalbert Wahl in seiner Gesamtdarstellung auch einigen nichtjüdischen Persönlichkeiten zu, für die er eine besondere persönliche Abneigung empfand. So porträtierte er etwa August Bebel als „Jünger des jüdischen Propheten "174. Hintergrund der Abneigung Wahls war unter anderem Bebels Schrift Die Frau und der Sozialismus (1879), die der Historiker mit einem auffallend ausführlichen, polemischen Verriss bedachte. ${ }^{175}$ Von einem „durch und durch kranken Denken“ Bebels war hier die Rede und von einem „tiefe[n] Ekel“,

169 Zur Karriere Strousbergs vgl. Borchert, Eisenbahnkönig; Roth, Sturz.

170 Craig, Geschichte, S. 84.

171 Wahl, Geschichte, Bd. 1, S. 448.

172 Ebd., S. $448 \mathrm{f}$.

173 Ebd., S. 449.

174 Wahl, Geschichte, Bd. 2, S. 609.

175 Vgl. ebd., S. 607-612. Wahl warf Bebel zum einen das Vorgaukeln eines wissenschaftlichen Anspruchs, zum anderen mangelnde Menschenkenntnis und ein bewusstes „Schwarz in Schwarz"-Malen (S.608) der tatsächlichen rechtlichen und gesellschaftlichen Stellung der Frau vor, eine Einstellung, in der eine prinzipielle Staatsfeindlichkeit Bebels zum Vorschein komme. Zu den Inhalten und der Rezeption von Bebels Schrift vgl. Lopes/Roth, Men's feminism; Weiß, Frauenfrage. 
mit der „man“ das Buch nach der Lektüre beiseitelege. ${ }^{176}$ Vor allem dass Bebel einen Zusammenhang zwischen der Lösung der Klassenfrage und jener der Frauenfrage postuliert hatte, konnte Wahl dem Mitbegründer der Sozialdemokratischen Arbeiterpartei nicht verzeihen. Im Vergleich zur Darstellung Laskers und Strousbergs fiel Wahls Schilderung Bebels letztlich aber weniger abschätzig aus trotz eines ellenlangen Registers vermeintlicher Versündigungen des berühmten Sozialdemokraten. Der Tübinger Historiker respektierte die „rednerische und agitatorische Begabung“ Bebels und dessen Fertigkeiten als „Führer der Massen"177.

Generell erachtete Wahl das Auftreten und Wirken genialer „Führer“ als das eigentliche Movens der Geschichte. ${ }^{178}$ Dementsprechend orientierten sich seine Hoffnungen auf eine „Rettung“ des deutschen Volks nach 1918 ganz an dem Auftritt eines neuen politischen „Führers“ - ein für die Weimarer Rechte charakteristischer Gedanke. ${ }^{179}$ Wahl forderte jedoch, dass es nicht bei dem rein passiven „Glauben“ bleiben dürfe, dass „zur rechten Zeit [...] der Führer eben kommen werde, von dem allein wir mit Recht die Rettung“ erhoffen. Vielmehr gelte es dem noch unbekannten „geborenen Führer“ aktiv

„[entgegenzu] arbeiten, auch um uns selbst darauf vorzubereiten, den echten Führer zu erkennen oder zu erfühlen, wenn er kommt, und ihm zu folgen. [...] So gilt es denn für uns, die Verhältnisse so zu gestalten, daß wir dazu beitragen können, den einmal erkannten Führer auch an die Spitze zu bringen, soweit es in unserer Macht steht ${ }^{\text {" } 180}$.

DeR „VÖLKISCHE GEDANKE“ IN DER DEUTSCHEN GeSCHICHTE - Noch ehe der erste Band seiner Deutschen Geschichte erschienen war, erhielt Wahl 1925 die Gelegenheit, die ideologischen Grundlagen seiner Geschichtsbetrachtung in der Broschüre Der völkische Gedanke und die Höhepunkte der neueren deutschen Geschichte darzulegen. Das Büchlein erschien im Pädagogischen Magazin des Hermann Beyer \& Söhne Verlags im thüringischen Langensalza, das während der 1920er und 1930er Jahre von zahlreichen völkischen Autoren frequentiert wurde ${ }^{181}$ und

176 Wahl, Geschichte, Bd. 2, S. 609, 611.

177 Ebd., S. 612.

178 Vgl. Wahl, Führertum, S. 26f.: Wahl wandte sich in der Broschüre gegen die ,gerade in letzter Zeit immer wieder“ auftauchenden Auffassungen, „wonach schließlich doch gerade die Massen die eigentlichen Träger des geschichtlichen Lebens und Werdens seien“.

179 Vgl. Sontheimer, Denken, S. 268-280; Schreiner, „Wann kommt der Retter Deutschlands?“.

180 Wahl, Führertum, S. 42 (Herv. i. Orig.).

181 Neben der Arbeit Adalbert Wahls erschienen im „Pädagogischen Magazin“ des Verlags an einschlägigen Publikationen unter anderem Max Wundts Was heißt völkisch? (1924, $\left.{ }^{3} 1925\right)$, Max Maurenbrechers Völkischer Geschichtsunterricht. Entwurf eines Lehrplanes (1925), Max Kochs Richard Wagners geschichtliche völkische Sendung. Zur Fünfzigjahr-Feier der Bayreuther Bühnenfestspiele (1927), Otmar Freiherr von Verschuers Sozialpolitik und Rassenhygiene (1928), Hermann Tögels Rasse, Volk und alttestamentlicher Religionsunterricht (1931), Wilhelm Fricks Bevölkerungs- und Rassenpolitik (1933), Wilhelm Karl zu Isenburgs Das Problem der Rassenreinheit (1933), Martin Staemmlers Rassenpflege und Schule (1933, $\left.{ }^{4} 1937\right)$, Achim Gerckes Rasseforschung und Familienkunde (1934), Joseph Goebbels' Rassenfrage und Weltpropaganda (1934, 21937), Arthur Gütts Leibesübungen im Dienst der Rassenpflege (1935, 21937) und H. J. Kuhns Arndt und Jahn als völkisch-politische Denker (1936). 
seinerzeit „breit gelesen“182 war. Zugleich war Wahls Broschüre Teil der Reihe Schriften zur politischen Bildung, die von der „dem Alldeutschen Verband nahe stehende[n] Gesellschaft Deutscher Staat"183 herausgegeben wurde. Wahl beschreibt in der Broschüre vier ausgewählte Ereignisse der deutschen Geschichte seit dem Ende des Mittelalters, denen er jeweils katalysatorische Wirkung für Entstehung und Verbreitung des „völkischen Gedankens“ zusprach. Eingangs räumte Wahl zwar ein, dass die deutsche Geschichte im Vergleich zu jener Englands und Frankreichs lange Zeit weniger „Höhepunkte“ aufgewiesen habe. Gleichsam als „Ersatz“ konnte das „junge Volk“ der Deutschen laut Wahl jedoch auf eine außerordentlich ereignisreiche jüngere Geschichte zurückblicken, in der sich „weit über irgend ein anderes Land hinaus“ Höhepunkte akkumuliert hätten - „leuchtender und glänzender“ als jene „der andern“"184. Um diese These zu illustrieren, zog Wahl vier denkbar konventionelle Beispiele heran: Erstens die Reformation, zweitens die Zeit Friedrichs des Großen, drittens die Befreiungskriege und viertens schließlich die Reichsgründung von 1871.

Um erläutern zu können, worin seiner Ansicht nach der „völkische“ Kern dieser vier Untersuchungsgegenstände bestand, bemühte sich Wahl zunächst um eine Definition des Begriffs „völkisch“. Dieser schien ihm „schwankend geworden“ zu sein und der „Erläuterung“ zu bedürfen - eine seinerzeit gängige und nicht unberechtigte Beobachtung, zumal sich nach dem Ende des Ersten Weltkriegs eine Vielzahl unterschiedlichster „politischer Kräfte, kulturkritischer Theorien und literarischer Richtungen“ als „programmatische[s] Schlagwort"185 auf den Begriff beriefen und damit die schon vor 1918 entstandene Begriffsverwirrung zusätzlich steigerten. ${ }^{186}$ Wahl wollte den Begriff „völkisch“ weder als bloßes Synonym für „national“ verstanden wissen, noch ausschließlich mit „antisemitisch“ gleichsetzen. Keiner dieser beiden Zugänge werde dem Begriff ganz gerecht. „Nationale“ Bestrebungen“, so Wahl, liefen stets auf eine „Bildung der Nation“ und „die möglichste Ausdehnung von Macht und Ruhm“ hinaus, „,völkische“ Bestrebungen“ zielten hingegen darauf ab, „überall - im Staat und auf allen Gebieten der Kunst - das Eigentümliche des eigenen Volkes zum Siege [zu] führen“. Die vier von ihm beschriebenen historischen „Höhepunkte“ zeichneten sich demnach im Kern dadurch aus, die „deutsche Art“ auf jeweils spezifische Weise vertieft zu haben - ohne dabei „nach ausländischem, besonders französischem Vorbild“ vorgegangen zu sein oder sich „fremden Geist, etwa dem semitischen“187, unterworfen zu haben.

Mit Blick auf die Reformation gestand Wahl zunächst ein, dass „Luthers Tat“ nicht „wesentlich von dem völkischen Gedanken bestimmt“ gewesen sei. Allerdings sei das Wirken des großen Reformators „in die Zeit eines sichtlichen Er-

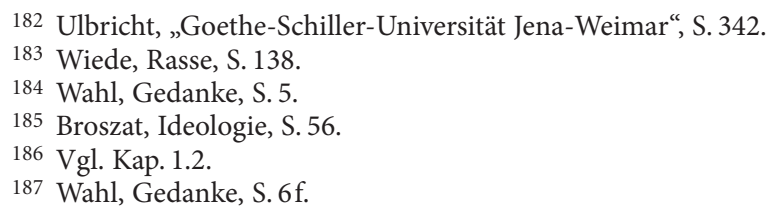


wachens völkischen Geistes“ gefallen. Überdies sei Luther in seinem Spätwerk vom „völkischen Gedanken stark berührt worden“188. Dieses Deutungsmuster war 40 Jahre zuvor in Heinrich von Treitschkes stark rezipierten Vortrag Luther und die deutsche Nation (1883) popularisiert worden ${ }^{189}$ und basierte nicht zuletzt auf den antijüdischen Verdikten in den späten Schriften Luthers. ${ }^{190}$ Auch Wahls Aussage, dass Luthers Entdeckung der „Art und [...] Vorzüge seines Volkes“ mit einer scharfen Wendung gegen die „Wälschen“ einhergegangen sei, entsprach der Denkschule Treitschkes. Mit der Behauptung, Luther habe infolge seines völkischen Erwachens gerade auch gegen „Polen oder sonstige Slaven“ Stellung bezogen, ging Wahl indes über sein großes Vorbild hinaus. Insgesamt betrachtet tritt Luther in Wahls Der völkische Gedanke und die Höhepunkte der neueren deutschen Geschichte als wegweisender Protagonist einer „völkische[n] Gesinnung“ in Erscheinung, „die nicht allein auf verstandesmäßigen Erwägungen“ beruht, sondern eine „Gesinnung der Liebe“ repräsentiert habe und daher gleichermaßen „warm“ und „blutvoll“ ausgefallen sei. ${ }^{191}$ Mit der Überordnung des Gemüts über den rein „rechnerischen" Verstand befand sich Wahl in der Tradition eines gegenaufklärerischen Antirationalismus, der sich zwischen den 1880er und 1930er Jahren insbesondere in der „Lebensphilosophie“ niederschlug. ${ }^{192}$

Dem Zeitalter Friedrichs des Großen als zweiten historischen „Höhepunkt“ ließ Wahl zunächst zwei „öde und sandige“ Jahrhunderte des „tiefen Darniederliegens des völkischen Bewußtseins“ vorangehen. Erst Friedrichs „Taten im Krieg“ und in der „äußere[n] und innere[n] Politik“ hätten diese überwunden. Den bekannten Sachverhalt, dass der preußische König der deutschen Kultur und Sprache mit offener Geringschätzung begegnete, verschwieg Wahl dabei nicht. Zur Wiedererweckung des völkischen Gedankens habe es daher bedeutender Mittler bedurft - gemeint waren hier vor allem zeitgenössische Schriftsteller. Diese hätten sich große Verdienste darin erworben, durch ihre patriotischen Beschreibungen die Taten und Leistungen Friedrichs ins rechte Licht zu rücken und das deutsche Volk mit ihnen vertraut zu machen. Erst durch die Kombination aus der faktischen Politik des Königs und den heroisierenden literarischen Darstellungen sei es gelungen, das „französische Joch abzuschütteln“193, wie es bis dahin auf dem deutschen Geist gelastet habe.

188 Ebd., S. 7f. (Herv. i. Orig.).

189 Vgl. Lehmann, „Er ist wir selber: der ewige Deutsche“.

190 Erinnert sei nur an die 1543 veröffentlichte Schrift Von den Juden und ihren Lügen. Einführend zu den judenfeindlichen Sentenzen im Spätwerk Luthers: Leppin, Luther, S. 340-344. $\mathrm{Zu}$ deren Rezeption unter völkischen Ideologen: Wiese, „Unheilsspuren“.

191 Wahl, Gedanke, S. 9-11; für weiterführende Hinweise zur völkisch-nationalistischen Lutherrezeption seit dem späten 19. Jahrhundert vgl. exemplarisch: Steigmann-Gall, „Furor protestanticus".

192 Vgl. Schnädelbach, Philosophie, S. 174-193. Der prominenteste Vertreter einer Lebensphilosophie in völkischer Verengung war August Julius Langbehn (1851-1907). Für dessen Gegenüberstellung von „Verstand“ und „Gemüth" vgl. Langbehn, Rembrandt, S. $302 \mathrm{f}$.

193 Wahl, Gedanke, S. 12 f. 
Die antinapoleonischen Befreiungskriege deutete Wahl wiederum primär als einen „Sieg im Reiche der Idee“. In diesem Fall galt Wahl das Aufleben völkischen Ideenguts weniger als Folge denn als „Vorbedingung des großen Aufschwungs“. Die Protagonisten der Befreiungskriege hatten sich nach dieser Lesart nicht nur in militärischem Sinne, sondern zugleich in ihrem ideellen Kampf gegen den „reine[n], naive[n] Individualismus“ der Französischen Revolution bewährt - ein Kampf, der laut Wahl als einer „des Guten gegen das Böse aufgefaßt werden“194 sollte. Diese außergewöhnlich triviale moralisierende Wertung entsprach dem damaligen Selbstverständnis der völkischen Bewegung als „Avantgarde einer breiten Abwehrfront gegen die ,Ideen von 1789“"195. Bereits 1913 hatte Wahl im Rahmen der aufwendigen Feierlichkeiten zum 100. Jahrestag der Völkerschlacht bei Leipzig die „Ideen von 1813“ ausgerufen und „als deutsches Gegenstück zu den Ideen der Französischen Revolution bezeichnet“196. Die Verfechter der „Ideen von 1789“ erklärte Wahl auch Mitte der 1920er Jahre zum ärgsten „Feind“ des deutschen Volks, hätten „ihre Staatsferne, ihr Individualismus, ihr materielles Glücksstreben, ihr Pazifismus und Kosmopolitismus“ doch den „kläglichen Zusammenbruch“ Preußens im Vorfeld der Befreiungskriege „verschuldet“197.

Zuletzt die Reichsgründung von 1871: Dass nicht schon während, sondern erst nach und infolge der Reichsgründung „völkische Stimmen“ laut geworden seien, führte Wahl auf die Einführung des „allgemeine[n] und gleiche[n] Wahlrecht[s] aller ,Individuen“" in der Verfassung des Deutschen Kaiserreichs vom 16. April 1871 zurück. Im Ganzen würdigte Wahl die Verfassung zwar als ein „Wunderwerk“, das in ihr fixierte Wahlrecht bezeichnete er jedoch als „häßlichen neuen Anbau [...] nach französischem Muster“, der gegen die natürliche Ungleichheit der Menschen ${ }^{198}$ verstoßen habe. Einen zentralen „Höhepunkt“ der deutschen Geschichte stellte die Reichsgründung in den Augen des Historikers trotzdem dar, zumal nach 1871 eine abermalige bewusste Wendung „zum völkischen Gedanken"199 in Deutschland vollzogen worden sei. Zum Beweis dieser Behauptung reihte Wahl eine willkürliche und eklektische Ahnengalerie auf, bestehend aus Richard Wagner, Wilhelm Leibl, Adolf Stoecker, Heinrich von Treitschke, Paul de Lagarde, Constantin Frantz und Hans von Wolzogen. Constantin Frantz etwa habe die Wendung zum völkischen Denken vollzogen, indem er „nach der Reichsgründung zusehends antisemitischer" geworden sei und „durchaus zutreffend“ vor einer „Judenherrschaft“ in Deutschland gewarnt habe. ${ }^{200}$ Auch die Entstehung der völkischen Bewegung im wilhelminischen Kaiserreich zog Wahl als

194 Ebd., S. 14f.

195 Ulbricht, „Ein heimlich offener Bund für das große Morgen...“, S. 67.

196 Bruendel, Volksgemeinschaft, S. 62.

197 Wahl, Gedanke, S. 16f.

198 Zum Glauben an die „natürliche Ungleichheit“ als wesentliches Charakteristikum völkischer Ideologie vgl. Breuer, Völkischen, S. 18f.

199 Wahl, Gedanke, S.23f. Keine Rede kann also davon sein, dass Wahl, wie Thomas Klein behauptet hat, in seiner Beurteilung der Reichsverfassung ,jegliche Problematik negiert" habe, vgl. Klein, Wahlprüfungsverfahren, S. XXII.

200 Wahl, Gedanke, S. 25-27. 
Beweis heran, wobei er sich jedoch mit ihr nicht gänzlich identifizierte und solidarisierte. So lehnte Wahl - ähnlich wie Wilhelm Stapel - insbesondere alle sogenannten ,arteigenen“, christentumsfeindlichen Religionsentwürfe strikt ab, die in Teilen der völkischen Bewegung seit dem ausgehenden 19. Jahrhundert vorgelegt und bisweilen auch vorgelebt worden waren. ${ }^{201}$ Mit Blick etwa auf den „ungesunden“ und „närrischen“ Glauben an die „nebelhafte Germanengottheit“ Wotan forderte Wahl die Völkischen dazu auf, sich von ihren „Schlacken“ zu reinigen. Auch die Tendenz zur maßlosen Selbstüberhöhung und die Neigung zur „gegenseitigen Befehdung“ müssten ablegt werden. Nur auf diese Weise, so Wahls Ratschlag, könne der „völkische Gedanke“ wie schon in der „Zeit der Freiheitskriege“ auferstehen und zum entscheidenden "Signal“ einer neuerlichen „Erhebung“ werden. ${ }^{202}$ 
